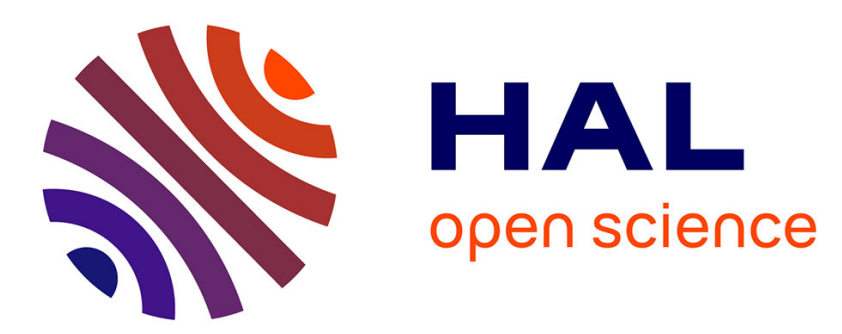

\title{
Numerical modelling of wave scattering by local inhomogeneities in elastic waveguides embedded into infinite media
}

\author{
Matthieu Gallezot, Fabien Treyssede, Laurent Laguerre
}

\section{To cite this version:}

Matthieu Gallezot, Fabien Treyssede, Laurent Laguerre. Numerical modelling of wave scattering by local inhomogeneities in elastic waveguides embedded into infinite media. Journal of Sound and Vibration, 2018, 31p. 10.1016/j.jsv.2018.11.044 hal-01942761

\section{HAL Id: hal-01942761 \\ https://hal.science/hal-01942761}

Submitted on 3 Dec 2018

HAL is a multi-disciplinary open access archive for the deposit and dissemination of scientific research documents, whether they are published or not. The documents may come from teaching and research institutions in France or abroad, or from public or private research centers.
L'archive ouverte pluridisciplinaire HAL, est destinée au dépôt et à la diffusion de documents scientifiques de niveau recherche, publiés ou non, émanant des établissements d'enseignement et de recherche français ou étrangers, des laboratoires publics ou privés. 


\title{
Numerical modelling of wave scattering by local inhomogeneities in elastic waveguides embedded into infinite media
}

\author{
Matthieu Gallezot ${ }^{\mathrm{a}, *}$, Fabien Treyssède ${ }^{\mathrm{a}}$, Laurent Laguerre ${ }^{\mathrm{a}}$ \\ ${ }^{a}$ IFSTTAR, GERS, GeoEND, F-44344, Bouguenais, France
}

\begin{abstract}
This paper proposes a numerical method to model wave scattering by local inhomogeneities in elastic open waveguides. The damaged zone of the waveguide and its vicinity are described by a finite-element model. This model is coupled on the cross-section boundaries to a modal representation of the field propagating along the waveguide axis in the undamaged parts, yielding transparent boundary conditions. However, open waveguides are unbounded in the transverse direction, which complicates both the numerical resolution and the physical analysis. Theoretically, the wave fields are described by a discrete sum of trapped modes and two continuous sums on radiation modes. The latter is sometimes approximated by a discrete sum of leaky modes, which grow at infinity in the transverse direction. In this paper, a Perfectly Matched Layer (PML) of finite thickness is introduced to absorb outgoing waves in the transverse direction, yielding three types of discrete modes: trapped modes, leaky modes and PML modes (PML modes oscillate mainly inside the layer and are non-intrinsic to the physics). Two numerical test cases are considered: the scattering at the junction between a closed and an open cylindrical waveguides and the scattering by an axisymmetrical notch. Good agreement with literature results is found. In particular, the influence of PML modes on the scattered field is highlighted through numerical tests. Due to the lack of power orthogonality of leaky modes, it is also shown that the modal cross power can become significant, which complicates the scattering analysis of open waveguides. Finally, the generality of the proposed method is discussed through a three-dimensional test case considering an embedded bar with an oblique break.
\end{abstract}

Keywords: waveguide ; leaky mode ; perfectly matched layer ; damage ; scattering ; finite element

\footnotetext{
${ }^{*}$ Corresponding author

Email address: matthieu.gallezot@ifsttar.fr (Matthieu Gallezot)
} 


\section{Introduction}

Elastic guided waves are often used for non-destructive evaluation (NDE) of elongated structures because they can propagate over a long distance while being sensitive to small damages. However, their scattering behaviour is difficult to interpret, because these waves are multi-modal and dispersive. Furthermore, when the structure is embedded into an infinite elastic medium (open waveguide), most of the waves are attenuated by radiation losses (leakage) into the surrounding medium, thus limiting the inspection range of NDE techniques.

In open waveguides, wave scattering by inhomogeneities has been studied experimentally for various structures such as steel bars [1] or seven-wires strands [2] embedded in cement grout, embedded rock bolts [3], reinforced bars [4] or pipes [5] in concrete. Yet, only a few numerical models have been developed, and they remain restricted to circular geometries (see Refs. [6-8]). Thus, this paper aims to propose a numerical model suitable for open waveguides of arbitrary cross-section with local inhomogeneities.

The idea is to describe the inhomogeneous (damaged) zone of the waveguide and its vicinity with a finite-element model. The latter is then coupled to a modal representation of the field propagating in the undamaged parts of the waveguide, yielding transparent boundary conditions. This principle has been widely applied in closed waveguides (i.e. in vacuum), see Refs. [9-15] for instance. In this paper, it will be referred to as hybrid method. Hybrid methods yield directly the reflection, transmission and conversion properties of the modes while remaining computationally fast. Furthermore, they are not badly affected by backward modes (with group and phase velocities of opposite signs), contrary to methods based on Perfectly Matched Layers (PML) truncating the undamaged parts along the waveguide axis [16].

Hybrid methods cannot be applied directly to open waveguides because the problem is unbounded in the transverse direction. The latter must be bounded for numerical purpose. Furthermore, the modal representation of waves strongly differs from closed waveguides.

In open waveguides, three types of modes can be distinguished: trapped modes, radiation modes and leaky modes. Trapped modes propagate without leakage attenuation along the waveguide axis. They are confined in the core of the waveguide or at the interface with the surrounding medium. Out of the core, these modes decay exponentially along the transverse direction. The existence of trapped modes depends on the contrast of materials between the core and the surrounding medium [17]. Radiation modes are standing waves along the transverse direction, which are propagative or evanescent in the axis direction [18-20]. Leaky modes propagate with leakage attenuation along the waveguide axis. However, these modes grow exponentially in the transverse direction $[6,18,20]$.

From a mathematical point of view, trapped modes are a discrete set of poles. Radiation modes are two continuous sets of modes (reduced to one in scalar waveguide), originating from branch cuts integration. Together, trapped modes and radiation modes form the modal basis of open waveguides: their 
superposition describes any wave field propagating in the waveguide [19, 21]. However, the continua of radiation modes are difficult to manipulate. They can be conveniently approximated by a discrete set of leaky modes in a zone restricted near the core $[20,22]$. In that case, leaky modes can provide key information such as the axial attenuation or the travelling velocity of wave packets [1].

From a numerical point of view, modes can be efficiently computed by discretizing only the cross-section of the waveguide while describing analytically the direction of wave propagation. To model open waveguides, a solution consists in using a PML of finite thickness in the transverse direction [23-26]. In this paper, this method will be called $P M L$ waveguide formulation. Other techniques have been proposed (in Refs. [27-31] for instance) and will not be discussed here. The PML waveguide formulation avoids most spurious reflections from the absorbing layer, which allows reducing its thickness. Furthermore, the modes are the solution of a linear eigenvalue problem.

As shown in Ref. [32] for scalar waveguides, it is noteworthy that the computation of leaky modes with a PML is mathematically relevant. Besides, radiation modes turn out to lie on two branch cuts rotated by the argument of the PML complex thickness [24] and discretized by the PML truncation to a finite thickness. These modes are often called PML modes (or Berenger modes). Although PML modes are non-intrinsic to the physics (they resonate mainly in the PML region), their modal superposition enables to accurately reconstruct the forced response of an elastic open waveguide [33]. Nevertheless, their contribution remains unclear as far as scattering problems are considered.

This paper proposes to use a PML in the transverse direction to truncate the surrounding medium in the finite element model of the inhomogeneous waveguide. This model is coupled to a modal representation based on the PML waveguide formulation, thus providing a hybrid method for open waveguides. Section 2 details the numerical method for three-dimensional waveguides of arbitrary cross-section and discusses the modal orthogonality properties specific to the PML formulation. In Sec. 3, numerical results are presented for three cases. First, the reflection at the junction between a closed and an open cylindrical waveguide is considered as a validation test case (Sec. 3.1). Results are also given for transmission. Then, the scattering of leaky modes by an axisymmetric notch is studied in low-frequency and high-frequency regimes (Sec. 3.2). The latter is of particular interest for NDE applications. The contribution of each type of modes to the scattered field is discussed. A particular attention is given to the PML mode contribution. Finally, the reflection of leaky modes by an oblique break is considered as an example of three-dimensional modelling.

\section{Numerical method}

\subsection{Weak form with a transverse PML}

Let us consider an elastic open waveguide made of a core of arbitrary cross-

section embedded into an infinite elastic medium. $z$ is the propagation axis of 


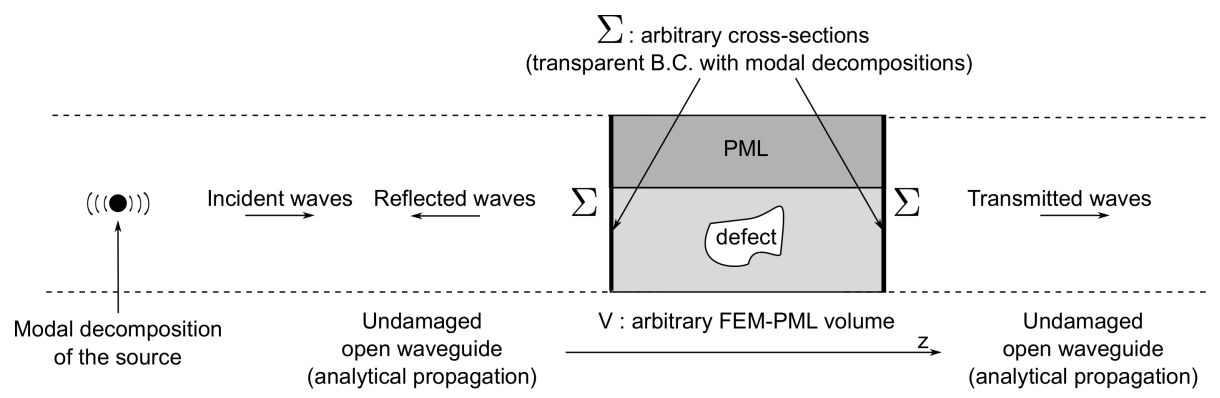

Figure 1: General description of a damaged open waveguide connected to semi-infinite undamaged open waveguides.

the waveguide. The waveguide contains a local inhomogeneity, such as a notch or a cross-section change for example. The domain $\tilde{V}$ is thus defined as a small part of the waveguide enclosing this inhomogeneity - see Fig. 1.

Small strains and displacements are assumed. A time-harmonic dependence $\mathrm{e}^{-\mathrm{j} \omega t}$ is chosen. In $\tilde{V}$, the weak formulation of elastodynamics is given by:

$$
\int_{\tilde{V}} \delta \tilde{\boldsymbol{\epsilon}}^{\mathrm{T}} \tilde{\boldsymbol{\sigma}} \mathrm{d} \tilde{V}-\omega^{2} \int_{\tilde{V}} \tilde{\rho} \delta \tilde{\mathbf{u}}^{\mathrm{T}} \tilde{\mathbf{u}} \mathrm{d} \tilde{V}=\int_{\tilde{V}} \delta \tilde{\mathbf{u}}^{\mathrm{T}} \tilde{\mathbf{f}} \mathrm{d} \tilde{V}+\int_{\partial \tilde{V}} \delta \tilde{\mathbf{u}}^{\mathrm{T}} \tilde{\mathbf{t}} \mathrm{d} \partial \tilde{V}
$$

where $\tilde{\boldsymbol{\epsilon}}$ and $\tilde{\boldsymbol{\sigma}}$ are the strain and stress vectors, $\tilde{\mathbf{u}}$ is the displacement vector, $\tilde{\mathbf{f}}$ is the vector of volumic forces inside $\tilde{V}$ and $\tilde{\mathbf{t}}$ is the traction vector applied on the surface $\partial \tilde{V}$. The subscript $\mathrm{T}$ denotes the matrix transpose. The stressstrain relation is given by $\tilde{\boldsymbol{\sigma}}=\tilde{\mathbf{C}} \tilde{\boldsymbol{\epsilon}}$, where $\tilde{\mathbf{C}}$ is the matrix of material properties (complex-valued for viscoelastic materials).

For numerical purpose, the infinite surrounding medium is truncated by a finite PML of thickness $h$. A radial PML is considered in this paper (a Cartesian PML could be used instead, see e.g. Ref. [25]). It is introduced by the analytic continuation [34] of the weak form (1) into the complex transverse coordinate $\tilde{r}$ :

$$
\tilde{r}(r)=\int_{0}^{r} \gamma(\xi) \mathrm{d} \xi
$$

$\gamma(r)$ is a user-defined complex-valued function for attenuating outgoing waves in the surrounding medium, such that:

- $\gamma(r)=1$ outside the PML region $(r<d)$,

- $\operatorname{Im}(\gamma(r))>0$ inside the PML region $(d<r<d+h)$.

$d$ is the position of the PML interface. An arbitrary boundary condition is applied at the end of the PML. In this paper, a Dirichlet condition is chosen.

Finally, Eq. (1) must be transformed to go back to the real radial direction $r$. The change of variable $\tilde{r} \mapsto r$ for any function $\tilde{g}(\tilde{r})$ yields:

$$
\tilde{g}(\tilde{r})=g(r), \mathrm{d} \tilde{r}=\gamma(r) \mathrm{d} r, \frac{\partial \tilde{g}}{\partial \tilde{r}}=\frac{\partial g}{\partial r} \frac{1}{\gamma(r)}, \mathrm{d} \tilde{V}=\frac{\tilde{r}(r) \gamma(r)}{r} \mathrm{~d} V .
$$




\subsection{Hybrid FE-modal approach}

A hybrid method is now proposed for the analysis of mode scattering by local inhomogeneities in elastic open waveguides.

The hybrid approach starts from the weak formulation (1) after applying the change of variable (3). The strain-displacement relation can be written as $\boldsymbol{\epsilon}=\mathbf{L u}$, where $\mathbf{L}$ is the operator including all spatial derivatives. A FE discretization can then be applied on the volume $V$. The displacement on each element is given by $\mathbf{u}^{e}(x, y, z, \omega)=\mathbf{N}_{V}^{e}(x, y, z) \mathbf{U}^{e}(\omega)$ where $\mathbf{N}_{V}^{e}(x, y, z)$ is the matrix of three-dimensional interpolation functions and $\mathbf{U}^{e}(\omega)$ is the vector of nodal displacements. Finally, the FE discretization leads to:

$$
\delta \mathbf{U}^{\mathrm{T}}\left(\mathbf{K}-\omega^{2} \mathbf{M}\right) \mathbf{U}=\delta \mathbf{U}^{\mathrm{T}} \mathbf{F},
$$

where $\mathbf{U}$ is the vector of displacements for all degrees of freedom (dofs) of $V, \mathbf{F}$ is the vector of forces, and $\mathbf{K}$ and $\mathbf{M}$ are the stiffness and mass matrices. The element matrices are given by:

$$
\mathbf{K}^{e}=\int \mathbf{N}_{V}^{e}{ }^{\mathrm{T}} \mathbf{L}^{\mathrm{T}} \mathbf{C} \mathbf{L} \mathbf{N}_{V}^{e} \frac{\tilde{r} \gamma}{r} \mathrm{~d} V, \mathbf{M}^{e}=\int \rho \mathbf{N}_{V}^{e}{ }^{\mathrm{T}} \mathbf{N}_{V}^{e} \frac{\tilde{r} \gamma}{r} \mathrm{~d} V .
$$

These matrices are complex-valued owing to the introduction of the PML.

$\mathbf{U}$ and $\mathbf{F}$ can be partitioned into the dofs belonging to the cross-section boundaries $\Sigma=\bigcup_{i} \Sigma_{i}$ (orthogonal to the waveguide axis $z$, see Fig. 1) and the remaining (i.e. internal) dofs, such that:

$$
\mathbf{U}=\left[\begin{array}{c}
\mathbf{U}_{\Sigma} \\
\mathbf{U}_{I}
\end{array}\right], \mathbf{F}=\left[\begin{array}{l}
\mathbf{F}_{\Sigma} \\
\mathbf{F}_{I}
\end{array}\right]
$$

where $I=V \backslash \Sigma$.

The main idea of the hybrid approach is to connect $V$ to semi-infinite homogeneous (i.e. inhomogeneity-free) waveguides through the boundaries $\Sigma$, thanks to transparent boundary conditions. This enables to greatly reduce the computational cost of the method. To do so, $\mathbf{U}_{\Sigma}$ and $\mathbf{F}_{\Sigma}$ are expanded on the wave modes of the homogeneous open waveguides as follows:

$$
\begin{aligned}
\mathbf{U}_{\Sigma} & =\sum_{n=1}^{N} \alpha_{-n} \mathbf{U}_{-n}+\sum_{n=1}^{N} \alpha_{n} \mathbf{U}_{n}, \\
\mathbf{F}_{\Sigma} & =\sum_{n=1}^{N} \alpha_{-n} \mathbf{F}_{-n}+\sum_{n=1}^{N} \alpha_{n} \mathbf{F}_{n} .
\end{aligned}
$$

$\mathbf{U}_{ \pm n}$ and $\mathbf{F}_{ \pm n}$ are the vectors of modal displacements and modal forces for a given mode $n$. These eigenvectors are computed with the PML waveguide formulation (briefly recalled in Sec. 2.3). The $\alpha_{ \pm n}$ are the modal coefficients. The subscript - denotes ingoing (incident) modes. The ingoing modal coefficients can be chosen to study the particular scattering behaviour of an incident 
mode or can represent a real source emitting somewhere in the undamaged waveguide (see Fig. 1). In the latter case, the ingoing modal coefficients are obtained from the modal decomposition of the forced response (see Ref. [33]), further propagated analytically and enforced to the appropriate cross-section boundary. The subscript + denotes outgoing (scattered) modes. $N$ denotes the number of modes retained in the modal expansions. Let us point out that the modal expansions (7) and (8) can be written for any arbitrary normalization of the mode shapes. Mode shapes can be conveniently normalized later during the post-processing steps — see Sec. 2.4.3.

It is convenient to gather known and unknown quantities into two different vectors $\mathbf{U}_{-}$and $\mathbf{U}_{+}$respectively defined as follows:

$$
\mathbf{U}_{-}=\left[\begin{array}{l}
\alpha_{-} \\
\mathbf{F}_{I}
\end{array}\right], \quad \mathbf{U}_{+}=\left[\begin{array}{l}
\alpha_{+} \\
\mathbf{U}_{I}
\end{array}\right] .
$$

$\alpha_{ \pm}$are the column vectors of modal coefficients, i.e. $\alpha_{ \pm}=\left[\begin{array}{llll}\alpha_{ \pm 1} & \alpha_{ \pm 2} & \ldots & \alpha_{ \pm N}\end{array}\right]^{\mathrm{T}}$. Combining Eq. (9) with Eqs. (7) and (8) gives:

$$
\mathbf{U}=\mathbf{G}_{u_{-}} \mathbf{U}_{-}+\mathbf{G}_{u_{+}} \mathbf{U}_{+}, \quad \mathbf{F}=\mathbf{G}_{f_{-}} \mathbf{U}_{-}+\mathbf{G}_{f_{+}} \mathbf{U}_{+},
$$

with the following matrices:

$\mathbf{G}_{u_{-}}=\left[\begin{array}{cc}\mathbf{B}_{u_{-}} & \mathbf{0} \\ \mathbf{0} & \mathbf{0}\end{array}\right], \quad \mathbf{G}_{u_{+}}=\left[\begin{array}{cc}\mathbf{B}_{u_{+}} & \mathbf{0} \\ \mathbf{0} & \mathbf{I}\end{array}\right], \quad \mathbf{G}_{f_{-}}=\left[\begin{array}{cc}\mathbf{B}_{f_{-}} & \mathbf{0} \\ \mathbf{0} & \mathbf{I}\end{array}\right], \quad \mathbf{G}_{f_{+}}=\left[\begin{array}{cc}\mathbf{B}_{f_{+}} & \mathbf{0} \\ \mathbf{0} & \mathbf{0}\end{array}\right]$,

where $\mathbf{B}_{u_{ \pm}}=\left[\begin{array}{llll}\mathbf{U}_{ \pm 1} & \mathbf{U}_{ \pm 2} & \ldots & \mathbf{U}_{ \pm N}\end{array}\right]$ and $\mathbf{B}_{f_{ \pm}}=\left[\begin{array}{llll}\mathbf{F}_{ \pm 1} & \mathbf{F}_{ \pm 2} & \ldots & \mathbf{F}_{ \pm N}\end{array}\right]$ are the basis of modal displacements and modal forces.

Using Eqs. (4) and (10) for any arbitrary field $\delta \mathbf{U}^{\mathrm{T}}=\left[\delta \alpha_{+}^{\mathrm{T}} \delta \mathbf{U}_{I}^{\mathrm{T}}\right]^{\mathrm{T}}$, one finally gets the following linear matrix system:

$$
\mathbf{G}_{u_{+}}^{\mathrm{T}}\left(\mathbf{D G}_{u_{+}}-\mathbf{G}_{f_{+}}\right) \mathbf{U}_{+}=\mathbf{G}_{u_{+}}^{\mathrm{T}}\left(\mathbf{G}_{f_{-}}-\mathbf{D G}_{u_{-}}\right) \mathbf{U}_{-},
$$

where $\mathbf{D}=\mathbf{K}-\omega^{2} \mathbf{M}$ is the dynamic stiffness matrix. The system (12) is solved for each angular frequency $\omega$, and yields the internal displacements of the FE domain as well as the scattered modal coefficients. From Eqs. (7) and (8), the total displacements and the forces can be obtained on the cross-section boundaries. They can be further propagated analytically to get the wave fields at any point of the undamaged waveguides connected to the FE domain (see Fig. 1).

\subsection{PML waveguide formulation for mode computation}

Eigenvectors used in the modal expansions (7) and (8) can be obtained with the so-called PML waveguide formulation. The derivation of the PML waveguide formulation starts from the weak form (1) after the change of variable (3), written on each cross-section $\Sigma_{i}$, dropping the source-term, and assuming an $\mathrm{e}^{\mathrm{j} k z}$ dependence for $\mathbf{u}\left(\mathrm{e}^{-\mathrm{j} k z}\right.$ for $\left.\delta \mathbf{u}\right) . k$ is the axial wavenumber. Hence, the strain-displacement relation can be written as:

$$
\boldsymbol{\epsilon}=\left(\mathbf{L}_{S}+\mathrm{j} k \mathbf{L}_{z}\right) \mathbf{u}
$$


where $\mathbf{L}_{S}$ is the operator including all terms but derivatives with respect to the $z$-axis, and $\mathbf{L}_{z}$ is the operator of $z$-derivatives.

A FE discretization is then be applied on the cross-section. The displacement on each element can be written $\mathbf{u}^{e}(x, y, \omega)=\mathbf{N}_{\Sigma}^{e}(x, y) \boldsymbol{\Phi}^{e}(\omega)$, where $\mathbf{N}_{\Sigma}^{e}(x, y)$ is the matrix of two-dimensional interpolation functions and $\boldsymbol{\Phi}^{e}(\omega)$ is the vector of nodal displacements. The following quadratic eigenvalue problem is finally obtained $[25,33]$ :

$$
\left(\mathbf{K}_{1}-\omega^{2} \mathbf{M}_{S}+\mathrm{j} k\left(\mathbf{K}_{2}-\mathbf{K}_{2}^{\mathrm{T}}\right)+k^{2} \mathbf{K}_{3}\right) \boldsymbol{\Phi}=\mathbf{0},
$$

where the element matrices are given by:

$$
\begin{aligned}
\mathbf{K}_{1}^{e} & =\int \mathbf{N}_{\Sigma}^{e}{ }^{\mathrm{T}} \mathbf{L}_{S}^{\mathrm{T}} \mathbf{C} \mathbf{L}_{S} \mathbf{N}_{\Sigma}^{e} \frac{\tilde{r} \gamma}{r} \mathrm{~d} S, \mathbf{K}_{2}^{e}=\int \mathbf{N}_{\Sigma}^{e}{ }^{\mathrm{T}} \mathbf{L}_{S}^{\mathrm{T}} \mathbf{C} \mathbf{L}_{z} \mathbf{N}_{\Sigma}^{e} \frac{\tilde{r} \gamma}{r} \mathrm{~d} S, \\
\mathbf{K}_{3}^{e} & =\int \mathbf{N}_{\Sigma}^{e \mathrm{~T}} \mathbf{L}_{z}^{\mathrm{T}} \mathbf{C} \mathbf{L}_{z} \mathbf{N}_{\Sigma}^{e} \frac{\tilde{r} \gamma}{r} \mathrm{~d} S, \mathbf{M}_{S}^{e}=\int \rho \mathbf{N}_{\Sigma}^{e \mathrm{~T}} \mathbf{N}_{\Sigma}^{e} \frac{\tilde{r} \gamma}{r} \mathrm{~d} S .
\end{aligned}
$$

$\boldsymbol{\Phi}$ is the vector of nodal displacements on the cross-section. More details can be found e.g. in Refs. [24, 25], as well as in Appendix A in the specific case of an axisymmetric circular open waveguide, which will be used to obtain the numerical results presented in Sec. 3.1 and 3.2.

The eigenproblem (14) must be linearized to make its resolution easier with standard eigensolvers [35]. The following linearized form is adopted:

$$
(\mathbf{A}-k \mathbf{B}) \mathbf{x}=\mathbf{0},
$$

with

$$
\mathbf{A}=\left[\begin{array}{cc}
\mathbf{0} & \mathbf{I} \\
\mathbf{K}_{1}-\omega^{2} \mathbf{M}_{S} & \mathrm{j}\left(\mathbf{K}_{2}-\mathbf{K}_{2}^{\mathrm{T}}\right)
\end{array}\right], \mathbf{B}=\left[\begin{array}{cc}
\mathbf{I} & \mathbf{0} \\
\mathbf{0} & -\mathbf{K}_{3}
\end{array}\right], \mathbf{x}=\left[\begin{array}{c}
\mathbf{\Phi} \\
k \boldsymbol{\Phi}
\end{array}\right] .
$$

Equation (16) is solved for each angular frequency $\omega$. Due to the symmetry of matrices $\mathbf{K}_{1}, \mathbf{K}_{3}$ and $\mathbf{M}_{S}$, the eigenspectrum is composed of pairs of eigensolutions, denoted $\left\{k_{n}, \mathbf{U}_{n}\right\}$ and $\left\{k_{-n}, \mathbf{U}_{-n}\right\}$ with $k_{-n}=-k_{n}$ for $(n=1, \ldots, N)$, representing $N$ outgoing modes and $N$ ingoing modes respectively [24, 25].

The modal forces $\mathbf{F}_{n}$ used in the modal expansion (8) are defined from [11, $36]$ :

$$
\int_{\Sigma_{i}} \delta \mathbf{u}^{\mathrm{T}} \mathbf{t}_{n}=\delta \boldsymbol{\Phi}^{\mathrm{T}} \mathbf{F}_{n}
$$

where for the mode $n, \mathbf{t}_{n}$ is the vector of the traction applied on the crosssection $\Sigma_{i}$ with an outward unit normal. It can be checked that $\mathbf{t}_{n}=\mathbf{L}_{z}^{\mathrm{T}} \boldsymbol{\sigma}_{n}=$ $\mathbf{L}_{z}^{\mathrm{T}} \mathbf{C}\left(\mathbf{L}_{S}+\mathrm{j} k \mathbf{L}_{z}\right) \mathbf{u}_{n}$, so that the modal forces are explicitly given by [11, 36]:

$$
\mathbf{F}_{ \pm n}=\left(\mathbf{K}_{2}^{\mathrm{T}}+\mathrm{j} k_{ \pm n} \mathbf{K}_{3}\right) \mathbf{U}_{ \pm n} .
$$




\subsection{Mode properties}

\subsubsection{Modal characteristics}

Modal characteristics can be readily computed from the eigensolutions of Eq. (14) and the finite element matrices (15) [25]. For a given mode $n$, its phase velocity is:

$$
v_{p_{n}}=\frac{\omega}{\operatorname{Re}\left(k_{n}\right)} .
$$

Its attenuation in $\mathrm{dB} \cdot \mathrm{m}^{-1}$ is:

$$
\eta_{n}=8.686 \operatorname{Im}\left(k_{n}\right) .
$$

The group velocity $v_{g_{n}}=\partial \omega / \partial k_{n}$ is computed as follows [25]:

$$
v_{g_{n}}=\operatorname{Re}\left[\left(\frac{2 \omega \mathbf{U}_{-n}^{\mathrm{T}} \mathbf{M} \mathbf{U}_{n}}{\mathbf{U}_{-n}^{\mathrm{T}}\left(\mathbf{j}\left(\mathbf{K}_{2}-\mathbf{K}_{2}^{\mathrm{T}}\right)+2 k_{n} \mathbf{K}_{3}\right) \mathbf{U}_{n}}\right)^{-1}\right] .
$$

In open waveguides, the energy velocity can be defined by [37]:

$$
\bar{v}_{e_{n}}=\frac{\operatorname{Re}\left(\bar{P}_{n}\right)}{\operatorname{Re}\left(\bar{T}_{n}\right)+\operatorname{Re}\left(\bar{V}_{n}\right)} .
$$

where for each mode $n, \bar{P}_{n}$ is the normal component of the Poynting vector integrated on the core cross-section, $\bar{T}_{n}$ is the core cross-section and time-averaged kinetic energy, and $\bar{V}_{n}$ is the core cross-section and time-averaged potential energy. These quantities can be post-processed from [25]:

$$
\begin{aligned}
\bar{P}_{n} & =\frac{-\mathrm{j} \omega}{2} \overline{\mathbf{U}}_{n}^{*}\left(\overline{\mathbf{K}}_{2}^{\mathrm{T}}+\mathrm{j} k_{n} \overline{\mathbf{K}}_{3}\right) \overline{\mathbf{U}}_{n}, \\
\bar{T}_{n} & =\frac{\omega^{2}}{4} \overline{\mathbf{U}}_{n}^{*} \overline{\mathbf{M U}}_{n}, \\
\bar{V}_{n} & =\frac{1}{4} \overline{\mathbf{U}}_{n}^{*}\left(\overline{\mathbf{K}}_{1}+\mathrm{j} k_{n} \overline{\mathbf{K}}_{2}-\mathrm{j} k_{n}^{*} \overline{\mathbf{K}}_{2}^{\mathrm{T}}+k_{n}^{*} k_{n} \overline{\mathbf{K}}_{3}\right) \overline{\mathbf{U}}_{n} .
\end{aligned}
$$

where the ${ }^{*}$ superscript denotes conjugate transpose and the overbar stands for the restriction to the dofs of the core of the cross-section. Note that the integration is usually restricted to the core cross-section in open waveguides because of the growth of leaky modes in the transverse directions. This restriction is quite arbitrary, but gives a reasonable approximation of the travelling velocities of wave packets $[1,37]$. In this paper, the calculation of $\bar{P}_{n}$ and $\bar{v}_{e_{n}}$ will only be used for post-processing steps (i.e. for dispersion curves and normalization of the scattering coefficients).

\subsubsection{Determination of the travelling direction}

The hybrid method presented in Sec. 2.2 requires determining the travelling direction of the modes. In elastic waveguides, the sign of the phase velocity (i.e. the sign of the real part of the wavenumber) does not necessarily give 
the travelling direction because of the existence of backward modes, which have phase and group velocities of opposite signs [38, 39]. The travelling direction of the modes is determined according to the following criterion [33]:

- the $\operatorname{sign}$ of $\operatorname{Im}\left(k_{n}\right)$ if $\operatorname{Im}\left(k_{n}\right) \neq 0$, such that if $\operatorname{Im}\left(k_{n}\right)>0$, the mode is a positive-going mode (towards $z>0$ ), and if $\operatorname{Im}\left(k_{n}\right)<0$ the mode is negative-going (towards $z<0$ ).

- the sign of $v_{g_{n}}$ if $k_{n} \in \mathbb{R}$.

The criterion is valid both for closed and open elastic waveguides. In closed waveguides, the case $k_{n} \in \mathbb{R}$ corresponds to propagating modes. In open waveguides, modes with real wavenumbers are somehow particular because they correspond to trapped modes. Trapped modes exist only when the shear velocity is greater in the embedding medium than in the core (except if longitudinal and shear waves couple into Stoneley waves [17]). Besides in viscoelastic media, modes with real wavenumbers never occur, because the wavenumbers are then always complex-valued. In that case, the sign of the imaginary part of the wavenumber naturally gives the direction of energy decay along the axis of the waveguide, which corresponds to the travelling direction of the waves (according to the $\mathrm{e}^{\mathrm{j} k z}$ convention chosen in this paper).

\subsubsection{Post-processing of scattering coefficients}

In this paper, reflection and transmission coefficients are defined by considering an incident mode $-n$ through the section $\Sigma_{1}$. The scattered modal coefficients $\alpha_{+n}$ are directly obtained by solving (12). Owing to leakage losses, their values depend on the positions $z_{i}$ of the cross-sections $\Sigma_{i}$. With the hybrid method, these positions are user-defined parameters and are hence arbitrary. To circumvent this problem, a reference position is chosen to post-process the scattering coefficients. In this paper, this reference position is set to the inhomogeneity position, i.e. $z=z_{\text {ref }}$ - see Figs. 2a and 7a for instance. As done for viscoelastic problems in closed waveguides [40, 41], this amounts to retropropagate the modal coefficients to the inhomogeneity position. Besides, the value of the coefficients $\alpha_{+n}$ depends on the arbitrary normalization of the mode shapes. For the physical analysis of results, it is convenient to normalize the mode shapes with their modal power as given by Eq. (24).

Hence, the reflection coefficient of a mode $m$ is defined on $\Sigma_{1}$ by:

$$
R_{m, n}=\frac{\left|\alpha_{+m} \mathrm{e}^{+\mathrm{j} k_{+m}\left(z_{\mathrm{ref}}-z_{1}\right)}\right| \sqrt{\left|\operatorname{Re}\left(\bar{P}_{+m}\right)\right|}}{\left|\alpha_{-n} \mathrm{e}^{+\mathrm{j} k_{-n}\left(z_{\mathrm{ref}}-z_{1}\right)}\right| \sqrt{\left|\operatorname{Re}\left(\bar{P}_{-n}\right)\right|}} .
$$

Similarly, the transmission coefficient of a mode $m$ is defined on $\Sigma_{2}$ by:

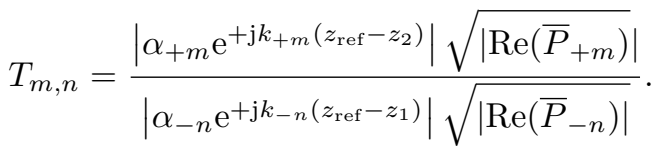


Such definitions enables to have scattering coefficients which are independent on the position of the cross-section $\Sigma_{i}$. Note that the square of Eqs. (27) and (28) yields readily the ratio of reflected and transmitted modal powers over the incident modal power respectively.

\subsection{Modal orthogonality in open waveguides}

In a waveguide, any arbitrary field can be expanded on a unique set of modes if they are orthogonal [38]. In open waveguides, orthogonality theoretically holds between trapped modes and radiation modes when integrated on the infinite cross-section [19, 21]. If the integration is restricted to the core cross-section, trapped and radiation modes are no longer orthogonal. On the other hand, leaky modes may approximate the contribution of radiation modes [20,22], but they cannot satisfy the orthogonality relationship since they grow to infinity in the transverse direction.

When the transverse problem is bounded by a finite PML, the authors have recently shown [33] that the following orthogonality relationship holds:

$$
Q_{m,-n}=\frac{\mathrm{j} \omega}{4}\left(\mathbf{U}_{m}^{\mathrm{T}} \mathbf{F}_{-n}-\mathbf{U}_{-n}^{\mathrm{T}} \mathbf{F}_{m}\right)=Q_{m,-m} \delta_{m n},
$$

This orthogonality relationship involves the whole cross-section of the waveguide (i.e. including the finite PML) and is applicable to any kind of modes without distinction (trapped, leaky and PML modes). It also remains valid for fully anisotropic or viscoelastic materials. Equation (29) guarantees the uniqueness of modal expansions (7) and (8). Actually, it is a discrete form of the socalled Auld's real orthogonality relationship [38], as already shown for closed viscoelastic waveguides [36].

It has to be emphasized that Eq. (29) is generally not a power orthogonality relationship. To prove this, let us start with the total time-averaged power flow across the whole cross-section, given by [11]:

$$
\Pi_{T}=\operatorname{Re}\left(-\frac{\mathrm{j} \omega}{2} \mathbf{U}_{\Sigma}^{*} \mathbf{F}_{\Sigma}\right) .
$$

Expanding the fields $\mathbf{U}_{\Sigma}$ and $\mathbf{F}_{\Sigma}$ on the modes yields (details are given in Appendix B):

$$
\Pi_{T}=\sum_{m=-N}^{N}\left|\alpha_{m}\right|^{2} \operatorname{Re}\left(P_{m}\right)+\sum_{m=-N}^{N} \sum_{n \neq m} \alpha_{n}^{*} \alpha_{m} P_{m, n},
$$

where $P_{m, n}=\frac{\mathrm{j} \omega}{4}\left(\mathbf{F}_{n}^{*} \mathbf{U}_{m}-\mathbf{U}_{n}^{*} \mathbf{F}_{m}\right)$ is the modal cross-power of modes $m$ and $n$. For $m=n$, one has $P_{m, m}=\operatorname{Re}\left(P_{m}\right)$, where $\operatorname{Re}\left(P_{m}\right)=\frac{\omega}{2} \operatorname{Im}\left(\mathbf{U}_{m}^{*} \mathbf{F}_{m}\right)$ is the modal power of the mode $m$. Note that $P_{m}$ is computed from the same equation as Eq. (24) but by removing the overbar in order to consider the whole cross-section.

There is so-called power orthogonality if the total power in the waveguide is the sum of the power propagated by each mode, that is if $P_{m, n}=0 \forall m \neq$ 


\begin{tabular}{cccccc}
\hline \hline Material & $\rho\left(\mathrm{kg} \cdot \mathrm{m}^{-3}\right)$ & $c_{l}\left(\mathrm{~m} \cdot \mathrm{s}^{-1}\right)$ & $c_{s}\left(\mathrm{~m} \cdot \mathrm{s}^{-1}\right)$ & $\eta_{l}\left(\mathrm{~Np} \cdot\right.$ wavelength $\left.^{-1}\right)$ & $\eta_{s}\left(\mathrm{~Np} \cdot\right.$ wavelength $\left.^{-1}\right)$ \\
\hline Steel & 7932 & 5960 & 3260 & 0.003 & 0.008 \\
Epoxy & 1170 & 2610 & 1000 & 0 & 0 \\
Cement grout & 1600 & 2810 & 1700 & 0.043 & 0.1 \\
\hline \hline
\end{tabular}

Table 1: Material properties

$n[38,42]$. However, the modal cross power $P_{m, n}$ is in general non-zero, except if the so-called Auld's complex biorthogonality relationship [38] is satisfied, that is to say, for real wavenumber modes only: in that case, $\mathbf{U}_{-n}^{\mathrm{T}}=\mathbf{U}_{n}^{*}$ and $\mathbf{F}_{-n}^{\mathrm{T}}=\mathbf{F}_{n}^{*}$ such that Eq. (29) becomes $Q_{m,-n}=P_{m, n}=\operatorname{Re}\left(P_{m}\right) \delta_{m n}[36]$.

As a consequence, there is no power orthogonality $\left(Q_{m,-n} \neq P_{m, n}\right)$ in open waveguides involving leaky modes or in lossy closed waveguides (wavenumbers are complex). The modal cross power should thus be considered. This has been studied in electromagnetic open waveguides, see e.g. Ref [18, 20, 43-45]. As for scattering analysis, this phenomenon is likely to complicate the interpretation of results in terms of modes. This will be illustrated in Sec. 3.2.3.

\section{Results}

\subsection{The junction test case}

As a first test case, the reflection of the fundamental longitudinal guided mode $\mathrm{L}(0,1)$ at the junction between a closed and an open cylindrical waveguide is computed with the hybrid method and compared to literature results. Results are also presented for the transmission coefficient and the role of PML modes in the solution of the scattering problem is investigated.

\subsubsection{Description}

One considers a cylindrical steel waveguide of radius $a=1 \mathrm{~mm}$ partly embedded in an infinite epoxy medium. Material properties are given in Table 1 (in the following, pure elastic materials are considered, i.e. the longitudinal and shear bulk wave attenuations $\eta_{l}$ and $\eta_{s}$ are equal to $0 \mathrm{~Np} \cdot$ wavelength $^{-1}$ ).

In Ref. [6], the reflection of the $\mathrm{L}(0,1)$ guided mode at the junction has been studied both with a mode-matching method and with a transient finiteelement model. The geometry of the problem is axisymmetric and the ingoing mode is longitudinal, such that there is no modal conversion towards flexural or torsional modes. The problem can then be solved with the axisymmetrical formulation of the hybrid method (see Appendix A), thus reducing the modal basis to longitudinal modes only. The computational domain $V$ is depicted in Fig. 2a. The inlet and outlet cross-sections boundaries $\Sigma_{1}$ and $\Sigma_{2}$ are set at equal distances $\left|z_{i}-z_{\text {ref }}\right|=0.25 a$ from the junction. A free surface condition is applied on the outer surface of the closed waveguide region $\left(z<z_{\text {ref }}, r=a\right)$ and on the inlet of the epoxy layer $\left(z=z_{\text {ref }}, a<r<a+h\right)$. The epoxy layer is bounded by a transverse PML. Following Ref. [25], the attenuation of the PML is chosen as parabolic and is given by: 


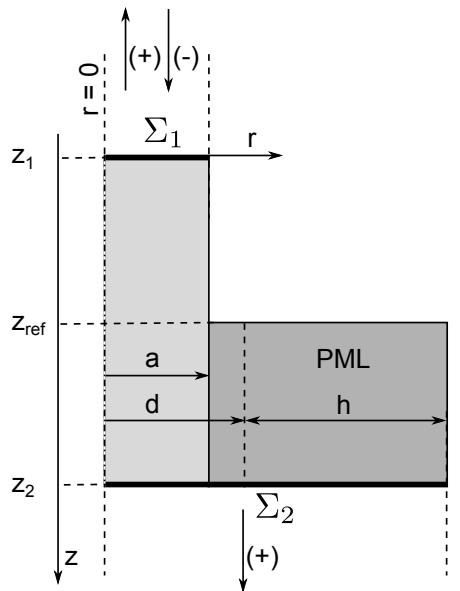

(a)

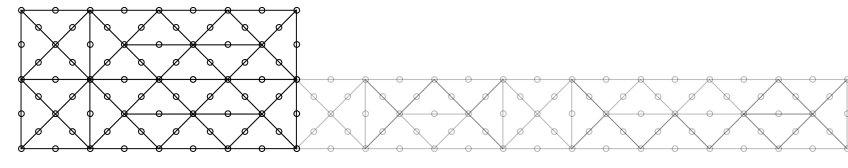

(b)

Figure 2: (Colour online) Junction test case. (a) Sketch of the computational domain $V$; (b) finite element mesh (black: physical domain, grey: PML domain with PML parameters $d=a$, $h=2 a)$.

$$
\gamma(r)= \begin{cases}1 & \text { if } r \leq d \\ 1+3(\hat{\gamma}-1)\left(\frac{r-d}{h}\right)^{2} & \text { if } r>d\end{cases}
$$

where $\hat{\gamma}=\frac{1}{h} \int_{d}^{d+h} \gamma(\xi) d \xi$ is the average value of $\gamma(r)$ inside the PML region. $\hat{\gamma}$ and $h$ are user-defined parameters. To avoid spurious eigenvalues and to enhance the attenuation of leaky modes, it is preferable to set the PML close to the core [32]. In the following, the distance is set to $d=a$ and the PML thickness is set to $h=2 a$. Note that in order to accurately compute leaky modes at the lowest frequencies (large wavelengths), the PML must have a relatively large complex thickness $d+\hat{\gamma} h$. After numerical tests, the value of $\hat{\gamma}$ has been set to $\hat{\gamma}=8+16 \mathrm{j}$. Despite this large complex thickness, the computational cost remains low because a coarse mesh is sufficient to obtain acceptable results in the frequency range of interest. The dispersion curves of the fundamental $\mathrm{L}(0,1)$ mode computed at both the inlet ant outlet cross-sections are given in Fig. 3 (PML modes have been filtered out from the visualization following the criterion of Appendix $\mathrm{C}$ ).

The domain $V$ is meshed with 6-nodes triangle elements, yielding a total of 338 dofs (see Fig. 2b) with 18 dofs on the cross-section $\Sigma_{1}$ and 52 dofs on the cross-section $\Sigma_{2}$. The latter values must be multiplied by two for the linearized eigenvalue problem (see Sec. 2.3). The eigenproblem (16) is solved with the ARPACK library [46]. Based on an implicit restarted Arnoldi method, this library enables to obtain a user-defined number of eigenvalues (denoted as $2 N$ ), corresponding to the closest in absolute value to a user-defined shift (set to 0 


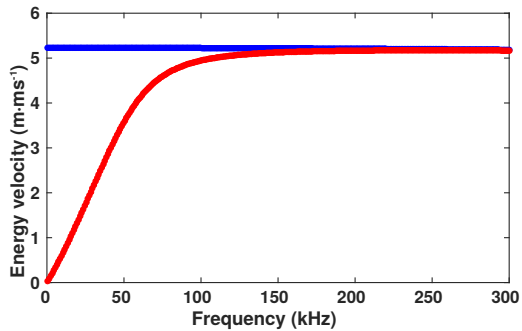

(a)

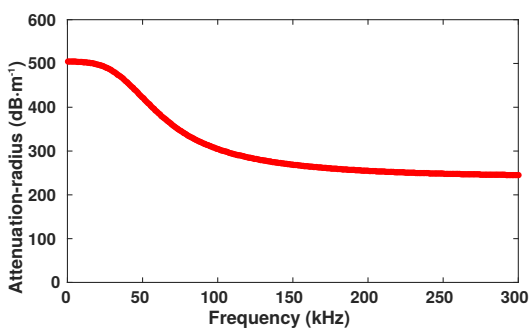

(b)

Figure 3: (Colour online) Dispersion curves obtained with the PML waveguide formulation. The PML parameters are $d=a, h=2 a, \hat{\gamma}=8+16 \mathrm{j}$ and the filtering criterion is $\eta_{\min }=0.5$. Blue dots: the $\mathrm{L}(0,1)$ guided mode in an elastic steel closed waveguide ; red dots: the $\mathrm{L}(0,1)$ leaky mode in a steel waveguide embedded into epoxy. Results for: (a) the energy velocity; (b) the attenuation.

in this paper). For this test case, $N=20$ modes are initially computed on each cross-section. The number of modes finally retained in the modal expansions is denoted $N_{\Sigma_{i}}(i=1,2)$.

\subsubsection{Numerical results}

Figure 4 shows the wavenumber spectrum computed on the cross-section $\Sigma_{2}$ (open waveguide), in the complex plane $(\operatorname{Re}(k a)>0, \operatorname{Im}(k a)>0)$ and at $f=300 \mathrm{kHz}$. The spectrum includes the $\mathrm{L}(0,1)$ leaky mode and $19 \mathrm{PML}$ modes. As expected, PML modes close to the real axis $(\operatorname{Im}(k a)=0)$ lie along two hyperbolas corresponding to branch cuts rotated with an angle $-\arg (d+h \hat{\gamma})$, and starting at branch points $\omega a / c_{l, s}\left(c_{l, s}\right.$ being the bulk velocities in the epoxy layer). A deviation from the hyperbolas can be observed far from the real axis. This deviation can be reduced by using a finer FE mesh [24]. Further details on the behaviour of PML modes can be found in Refs. [25, 33]. As far as the crosssection $\Sigma_{1}$ is concerned (closed waveguide), the wavenumber spectrum includes the $\mathrm{L}(0,1)$ guided mode (real wavenumber) and 19 complex inhomogeneous or evanescent modes (spectrum not shown for conciseness).

First, all the modes computed on $\Sigma_{2}$ are retained in the modal expansion $\left(N_{\Sigma_{2}}=N=20\right)$. On $\Sigma_{1}$, it has been checked that the inclusion of evanescent or inhomogeneous modes has a negligible influence on the results (in agreement with Ref. [6]). Hence, only the $\mathrm{L}(0,1)$ guided mode is retained in the modal expansions $\left(N_{\Sigma_{1}}=1\right)$. The reflection coefficient is shown in Fig. 5. A very good agreement is obtained with Ref. [6]. Comparisons have been also made with other results of Ref. [6] for various radii and material properties and have shown a similar accuracy of results (not shown). The transmission coefficient of the $\mathrm{L}(0,1)$ mode has also been computed with the hybrid method (Fig. 6). As can be observed, the transmission curve exhibits an opposite behaviour compared to the reflection curve and quickly tends to one as the frequency increases.

Second, only the leaky mode is retained in the modal expansion on $\Sigma_{2}$ 


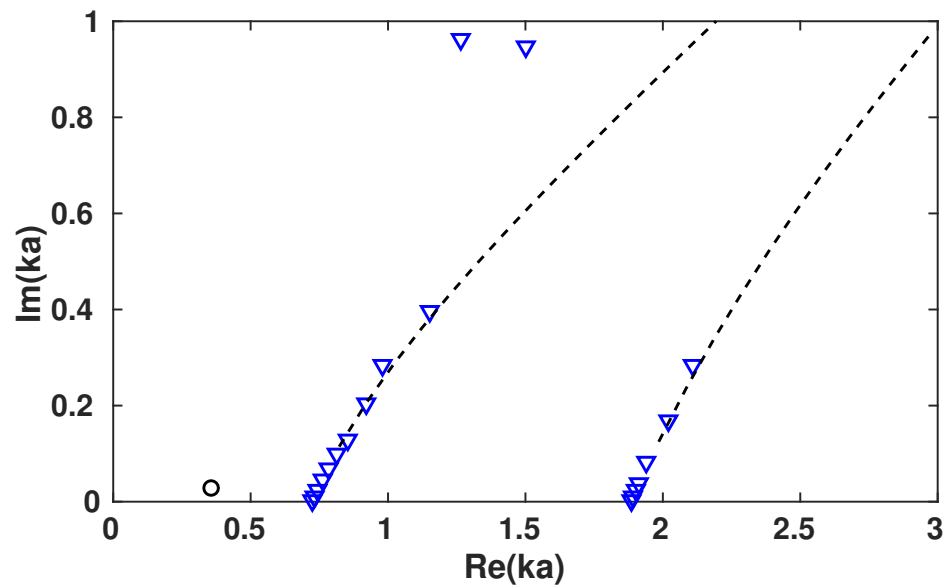

Figure 4: (Colour online) Wavenumber spectrum $(N=20)$ of an elastic steel waveguide embedded into epoxy computed with the PML waveguide formulation (cross-section $\Sigma_{2}$ ), at $f=300 \mathrm{kHz}$. Black circle: leaky mode, blue triangles: PML modes, dashed black lines: theoretical branch cuts with a finite PML rotated by $-\arg (d+h \hat{\gamma})$. PML parameters: $d=$ $a, h=2 a, \hat{\gamma}=8+16 \mathrm{j}$.

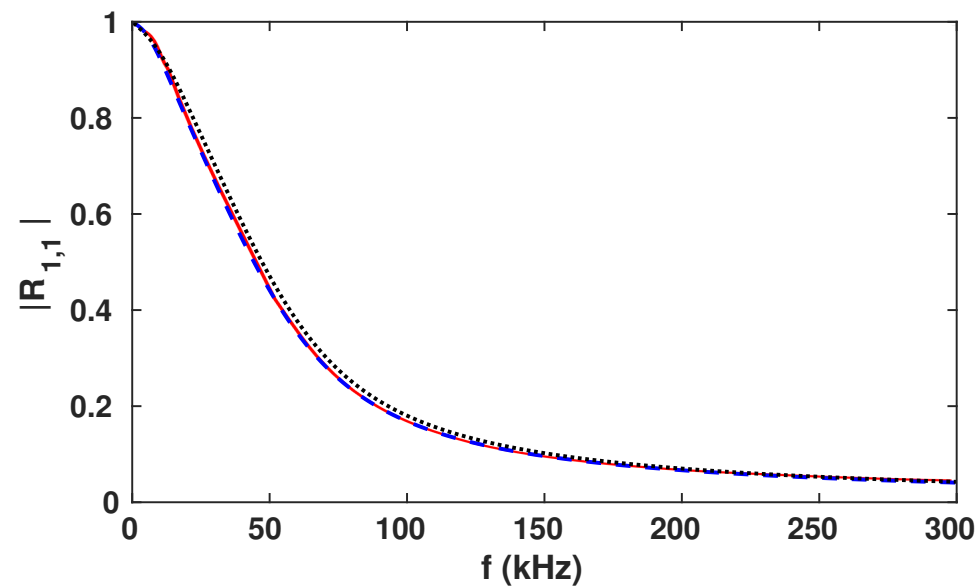

Figure 5: (Colour online) Reflection coefficient of the $\mathrm{L}(0,1)$ mode by the junction. Red curve: results of Ref. [6]. Blue dashed curve: hybrid approach with $N_{\Sigma_{1}}=1, N_{\Sigma_{2}}=20$. Black dotted curve: hybrid approach with $N_{\Sigma_{1}}=1, N_{\Sigma_{2}}=1$. PML parameters: $d=a$, $h=2 a, \hat{\gamma}=8+16 \mathrm{j}$. 


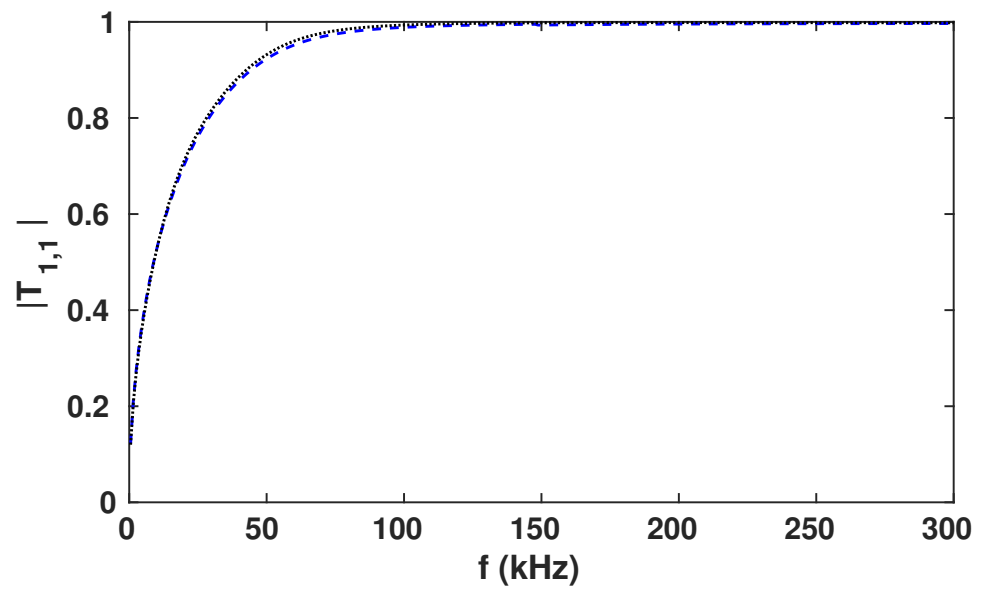

Figure 6: (Colour online) Transmission coefficient of the $\mathrm{L}(0,1)$ leaky mode by the junction. Blue dashed curve: hybrid approach with $N_{\Sigma_{1}}=1, N_{\Sigma_{2}}=20$. Black dotted curve: hybrid approach with $N_{\Sigma_{1}}=1, N_{\Sigma_{2}}=1$. PML parameters: $d=a, h=2 a, \hat{\gamma}=8+16 \mathrm{j}$.

$\left(N_{\Sigma_{2}}=1\right)$. As shown in Fig. 4, some PML modes close to the branch points have a quite low attenuation (given by $\operatorname{Im}(k a)$ ), smaller than the attenuation of the $\mathrm{L}(0,1)$ leaky mode. Hence, their contribution to the solution could $a$ priori be significant. Yet, the contribution of PML modes turns out to be weak and a good approximation of the scattering by the junction is still obtained with the leaky mode only (see Figs. 5 and 6). Actually, PML modes oscillate mainly inside the PML layer, which significantly reduces their contribution to the solution inside the core.

It is noteworthy that, in the very far-field, the contribution of PML modes can become predominant over leaky modes due to long-term diffraction (longterm diffraction decays geometrically, as opposed to leaky modes which decay exponentially). This has been demonstrated for the forced response of waveguides in Refs. [33, 47]. In our scattering problem, this could be also observed if an artificially large distance from the junction were chosen. Nevertheless, the goal of hybrid methods is to reduce the FE mesh close to the vicinity of the inhomogeneity. Therefore, in practice, the distance $\left|z_{i}-z_{\text {ref }}\right|$ is expected to remain low enough to prevent the influence of long-term diffraction.

As will be shown for the next test case, including PML modes can yet be necessary to accurately reconstruct the scattered solution in the very near field, i.e. when the cross-section boundaries are set very close to the inhomogeneity.

\subsection{Scattering by an axisymmetric notch}

A cylindrical open waveguide damaged by a notch in the core is now considered. The scattering analysis is performed both in a low and a high-frequency regime. The contribution of PML modes to the solution is discussed in the near 

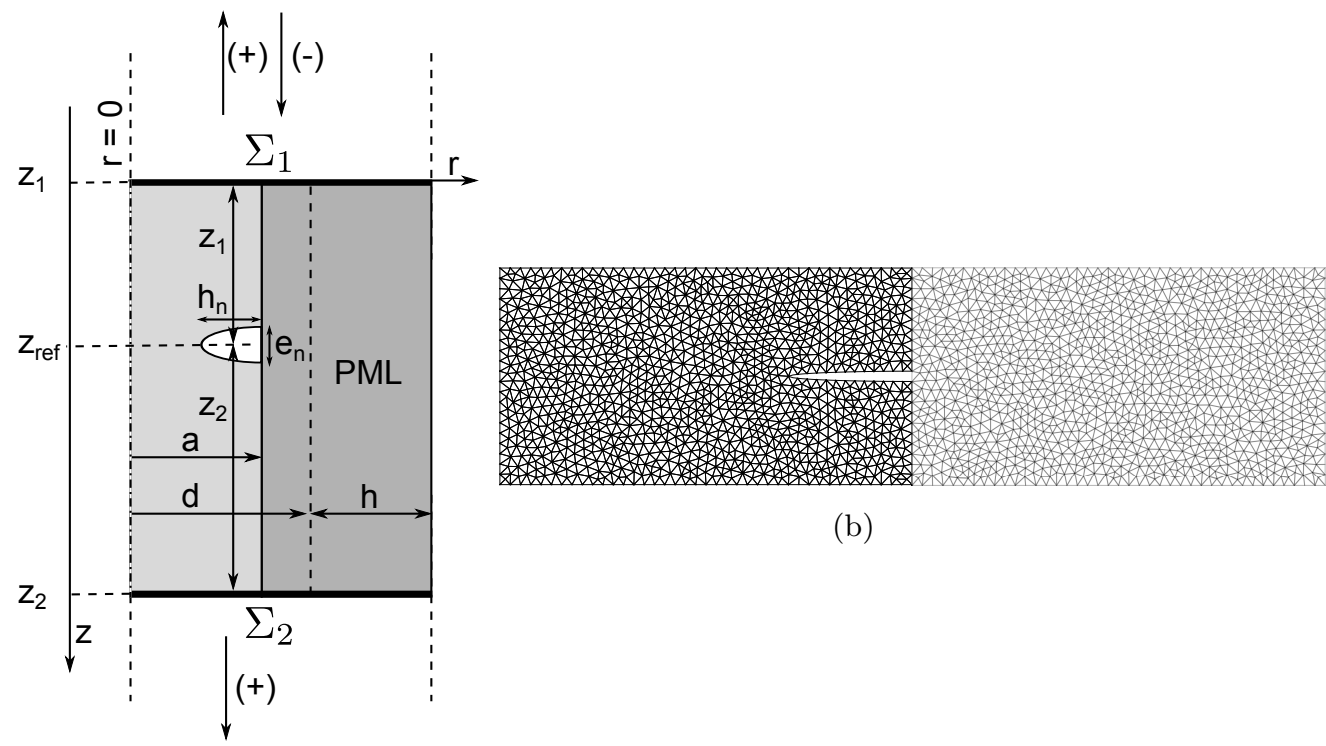

(b)

(a)

Figure 7: (Colour online) Notch test case. (a) Scheme of the computational domain $V$; (b) finite element mesh for $\left|z_{i}-z_{\text {ref }}\right|=0.25 a$ (black: physical domain for a notch of width $e_{n} / a=0.025$ and depth $h_{n} / a=0.3$; grey: PML domain with PML parameters $d=h=a$ ).

field. This test case also enables to consider a configuration with large modal cross-powers, highlighting their influence on the modal scattering coefficients.

\subsubsection{Description}

Let us consider a cylindrical waveguide made of viscoelastic steel and embedded into an infinite cement grout medium. Material properties are given in Table 1 . The core of the waveguide has a radius $a=10 \mathrm{~mm}$ and is damaged by a notch described by a semi ellipse centred at $\left(r=a, z=z_{\text {ref }}\right)$, of depth $h_{n}$ along $r$ and width $e_{n}$ along $z$ (see Fig. 7a). The width of the notch is set to $e_{n} / a=0.025$ and the depth $h_{n} / a$ is a varying parameter.

For simplicity, one assumes an axisymmetric notch (the notch is hence of annular type), so that the problem remains fully axisymmetric. Besides, the study is limited to the scattering of longitudinal modes only (torsional and flexural modes are not considered). As in Sec. 3.1, the problem can then be solved with an axisymmetrical formulation of the hybrid method. The computational domain is depicted in Fig. 7a. The embedding medium is truncated with a PML of same attenuation profile than in Eq. (32).

Dispersion curves of the waveguide are shown in Fig. 8 for 12 modes corresponding to leaky longitudinal modes, conventionally labelled as $\mathrm{L}(0, n)$. PML modes have been filtered out from the visualization following the criterion of Appendix C. Because of material properties, no trapped modes can propagate. The results of Ref. [1] calculated by a global matrix approach are also shown, 


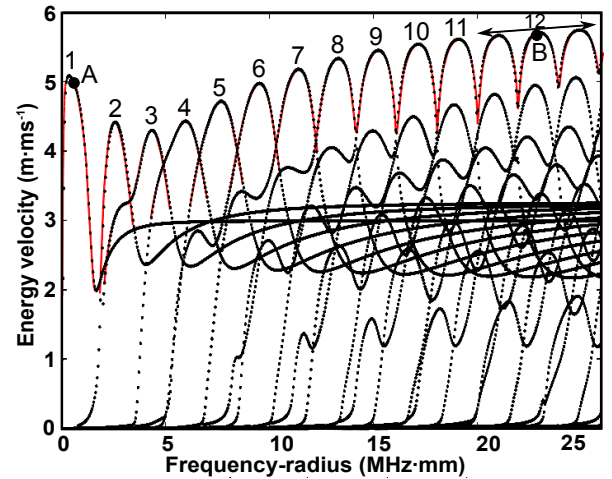

(a)

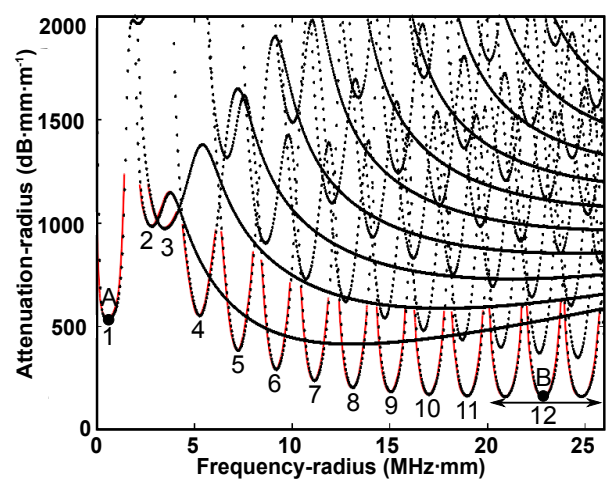

(b)

Figure 8: (Colour online) Dispersion curves of a viscoelastic steel cylinder embedded into viscoelastic cement grout obtained with the PML waveguide formulation (black dots). The PML parameters are $h=a, d=a, \hat{\gamma}=1+2 \mathrm{j}$ and the filtering criterion for PML modes is $\eta_{\min }=0.6$. Red lines: results of Ref. [1]. Point $\mathrm{A}$ indicates $\mathrm{f}=0.6 \mathrm{MHz} \cdot \mathrm{mmand}$ Point $\mathrm{B}$ indicates $\mathrm{f}=22.89 \mathrm{MHz} \cdot \mathrm{mm}$. Results for: (a) the energy velocity $\bar{v}_{e}$; (b) the attenuation.

which confirms the accuracy of leaky mode computation with the PML waveguide formulation. The following PML parameters have been used: $h=a, d=a$, $\hat{\gamma}=1+2 \mathrm{j}$.

In open waveguides at high frequencies, the energy can be concentrated into the core, thus reducing the leakage attenuation [1]. Herein, the minimal attenuation is equal to $159 \mathrm{~dB} \cdot \mathrm{mm} \cdot \mathrm{m}^{-1}$ and is reached for the 12 th mode $(\mathrm{L}(0,12))$ at point $\mathrm{B}(\mathrm{f}=22.89 \mathrm{MHz} \cdot \mathrm{mm})$ - see Fig. $8 \mathrm{~b}$. The attenuation of higher order modes is greater because of the predominance of viscoelasticity over leakage for higher frequencies. Since the 12th mode propagates over greater distances than the others, it is quite attractive for NDE applications. Hence, it is interesting to get a better understanding of its interaction with inhomogeneities.

In the following, the scattering of the 12th leaky mode by a notch of varying depth $h_{n}$ is studied. The problem is solved over the second lobe (between 21.64 $\mathrm{MHz} \cdot \mathrm{mmand} 24.12 \mathrm{MHz} \cdot \mathrm{mm}$ ) and particularly at point B. For the sake of comparison, the reflection and transmission of the 1st leaky mode $(\mathrm{L}(0,1))$ at $f=0.6 \mathrm{MHz} \cdot \mathrm{mm}$ (point A in Fig. 8) is also computed. Both reflection and transmission coefficients are post-processed at the position $z_{\text {ref }}$ according to Eqs. (27) and (28).

The inlet and outlet cross-sections boundaries, $\Sigma_{1}$ and $\Sigma_{2}$, are set at distances $\left|z_{i}-z_{\text {ref }}\right|=a$ for the low-frequency regime (point A) and $\left|z_{i}-z_{\text {ref }}\right|=0.25 a$ for the high-frequency regime. The domain is meshed with 6 -nodes triangle elements, yielding about 53000 dofs and 18000 dofs respectively, with 324 dofs on both cross-sections - see Fig. 7b. It has been checked that the contribution of PML modes was negligible. Therefore, only leaky modes are retained yielding $N_{\Sigma_{1}}=N_{\Sigma_{2}}=13$ at point $\mathrm{A}$ and $N_{\Sigma_{1}}=N_{\Sigma_{2}}=40$ at point B. Results are presented in Sec. 3.2.2 and 3.2.3. 


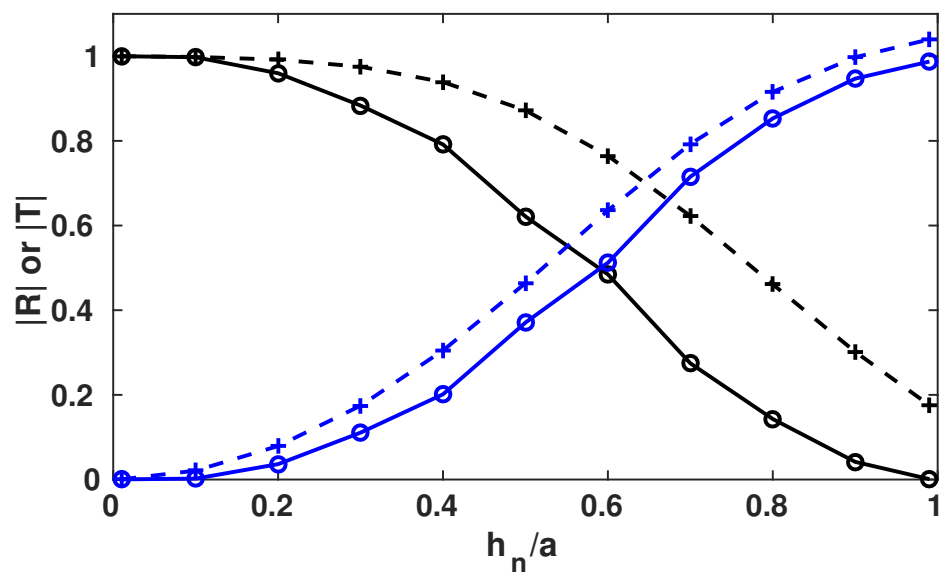

Figure 9: (Colour online) Scattering by a notch of varying depth. Dashed cross lines: $\left|R_{1,1}\right|$ (blue) and $\left|T_{1,1}\right|$ (black) at point $\mathrm{A}(f=0.6 \mathrm{MHz} \cdot \mathrm{mm})$, with $\left|z_{i}-z_{\text {ref }}\right|=a$ and $N_{\Sigma_{1}}=$ $N_{\Sigma_{2}}=13$. Solid lines: $\left|R_{12,12}\right|$ (blue) and $\left|T_{12,12}\right|$ (black) at point B $(f=22.89 \mathrm{MHz} \cdot \mathrm{mm})$ with $\left|z_{i}-z_{\text {ref }}\right|=0.25 a$ and $N_{\Sigma_{1}}=N_{\Sigma_{2}}=40$. PML parameters: $d=h=a, \hat{\gamma}=1+2 \mathrm{j}$.

To highlight the contribution of PML modes in the very near-field of the notch, another configuration is considered at point A by reducing the crosssection distance to $\left|z_{i}-z_{\text {ref }}\right|=0.25 a$. Results are presented in Sec. 3.2.4.

\subsubsection{Results}

Reflection and transmission coefficients are displayed in Fig. 9 for various notch depths, at point A for the 1st leaky mode and at point B for the 12th leaky mode. The coefficients exhibit the same general trend at both frequencies. The reflection coefficient increases when the notch depth increases, and conversely for the transmission coefficient. Note that the reflection coefficient at point A for a notch of depth $h_{n} / a>0.9 a$ is greater than one (this phenomenon is discussed further). It is noteworthy our results are very close to those of Ref. [48], obtained with the $\mathrm{L}(0,1)$ mode in the low-frequency regime with a transient finite-element model for an elastic steel core embedded into grout and damaged by a rectangular notch. This similarity confirms the reliability of the proposed hybrid method for the scattering analysis of open waveguides.

The differences in the scattering behaviour between low and high frequencies, as observed in Fig. 9, can be related to the distribution of the mode energy over the cross-section. For small depth notches, the reflection of the 12th leaky mode is lower than for the 1st one because its energy is more concentrated at the centre of the core of the waveguide. On the other hand, it can be observed that some energy of the 1st leaky mode is still transmitted for a full-depth notch $\left(\left|T_{1,1}\right|=\right.$ 0.18 ), indicating an energy transfer between both sides of the notch through the embedding medium (also in agreement with Ref. [48]). This is not the case for the 12th leaky mode, which transmission coefficient is negligible. The scattered 


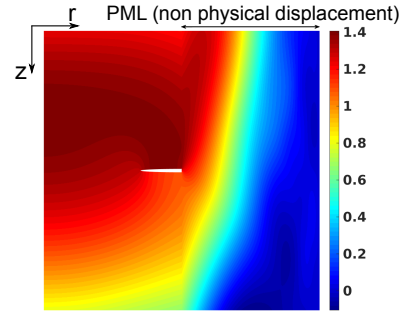

(a)

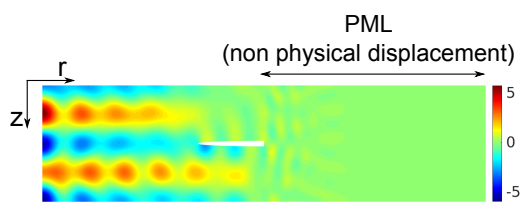

(b)

Figure 10: (Colour online) Scattered field $\operatorname{Re}\left(\mathbf{U}_{z} / a\right)$ by a notch of depth $h_{n} / a=0.3$, with the same model parameters as in Fig. 9: (a) at point A $(f=0.6 \mathrm{MHz} \cdot \mathrm{mm})$; (b) at point B $(f=22.89 \mathrm{MHz} \cdot \mathrm{mm})$.

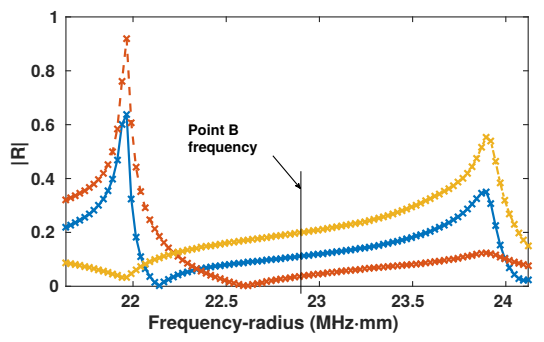

(a)

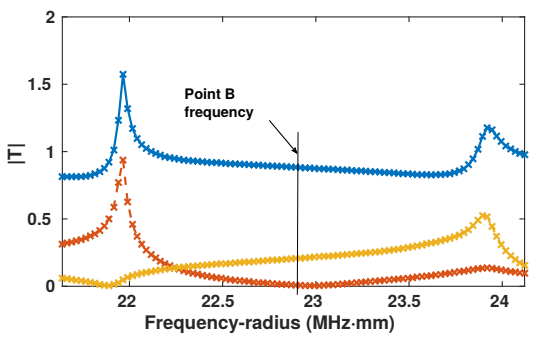

(b)

Figure 11: (Colour online) Scattering coefficients of the 12th, 13th and 14th leaky modes around point B. PML parameters: $d=h=a, \hat{\gamma}=1+2 \mathrm{j}$; notch depth $h_{n} / a=0.3$. (a) Reflection coefficients $\left|R_{12,12}\right|$ (blue line), $\left|R_{13,12}\right|$ (red dashed line), $\left|R_{14,12}\right|$ (yellow dashed dotted line); (b) transmission coefficients $\left|T_{12,12}\right|$ (blue line), $\left|T_{13,12}\right|$ (red dashed line), $\left|T_{14,12}\right|$ (yellow dashed dotted line).

fields computed at both frequencies are shown in Fig. 10. Differences of energy distribution over the cross-section can be clearly observed.

Figure 11 displays the reflection and transmission coefficients of several leaky modes at high frequencies around point B. Despite a high number of propagating modes, mode conversion is negligible except for a limited number of modes, namely the $\mathrm{L}(0,13)$ and mostly the $\mathrm{L}(0,14)$ leaky mode. Yet, it must be kept in mind that the scattering coefficients shown have been retropropagated at the inhomogeneity position. The propagation far from the inhomogeneity will favour the less attenuated mode, i.e. the 12th leaky mode here. In addition, the 12th leaky mode benefits from a high reflection coefficient, particularly for deep notches (see Fig. 9), which confirms the high reflection capability of high order low-leakage modes sometimes mentioned in the literature [49].

In Fig. 11, it should be noted that the reflection and transmission coefficients vary in a counterintuitive way. Indeed, instead of having opposite behaviour as normally expected, both coefficients curves sharply peak around 22 


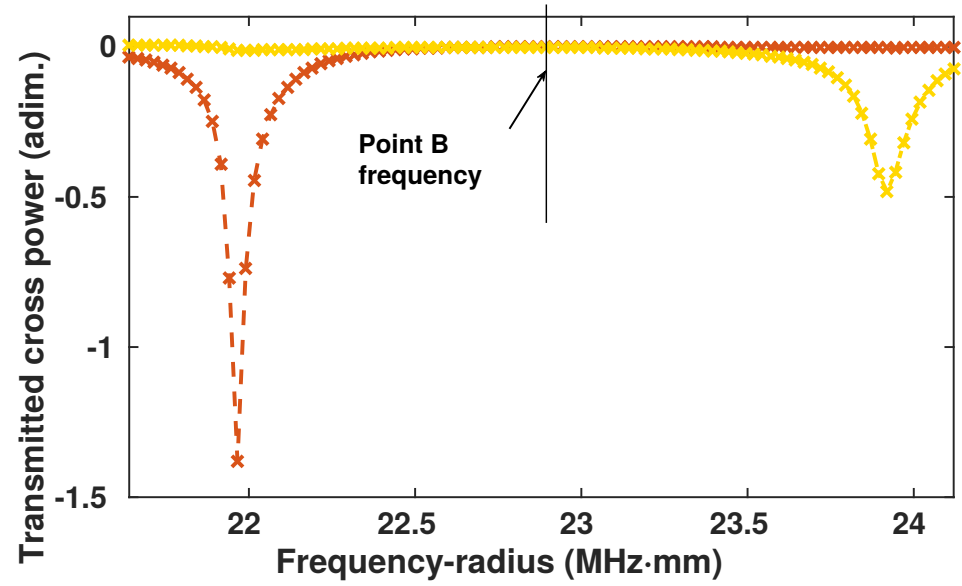

Figure 12: (Colour online) Transmitted cross power $P_{12,13}$ (red dashed line) and $P_{12,14}$ (yellow dashed dotted line). PML parameters: $d=h=a, \hat{\gamma}=1+2 \mathrm{j}$. Notch: $e_{n} / a=0.025, h_{n} / a=$ 0.3 .

$\mathrm{MHz} \cdot \mathrm{mmand} 24 \mathrm{MHz} \cdot \mathrm{mm}$. Furthermore, the transmission coefficient of the 12 th mode takes values greater than one (as already observed in Fig. 9 for the reflection of the 1st leaky mode at point A). Actually, these results cannot be analysed without further considerations on the modal cross power, as discussed in the next section.

\subsubsection{Considerations on the modal cross power}

Reflection and transmission coefficients are defined for each mode separately, and thus do not account for the modal cross power. The transmitted cross powers $P_{12,13}$ and $P_{12,14}$ are displayed in Fig. 12 at high frequencies (results are similar in reflection). Their values are negligible (particularly at point B) except around $22 \mathrm{MHz} \cdot \operatorname{mmand} 24 \mathrm{MHz} \cdot \mathrm{mm}$, i.e. at frequencies corresponding to the peaks previously observed in Fig. 11.

As shown in Fig. 13, one can observe that this phenomenon takes place at frequencies where mode crossing of phase velocity curves occurs. As noticed in Ref. [1] at high frequencies, the phase velocity curve of the 12th mode crosses those of the 13th and 14th modes. At these crossing frequencies, the modes have the same values of $\operatorname{Re}(k a)$ and similar mode shapes, which explains their strong interaction.

Note that such a mode crossing behaviour can also be observed in viscoelastic waveguides, as mentioned e.g. in Refs. [50, 51]. Hence, a similar phenomenon is likely to occur in the scattering analysis of closed but lossy waveguides (as already discussed in Sec. 2.5, the lack of power orthogonality also concerns viscoelastic waveguides).

These results show that when there is no power orthogonality relationship between modes, an interpretation of results based solely on the individual scat- 


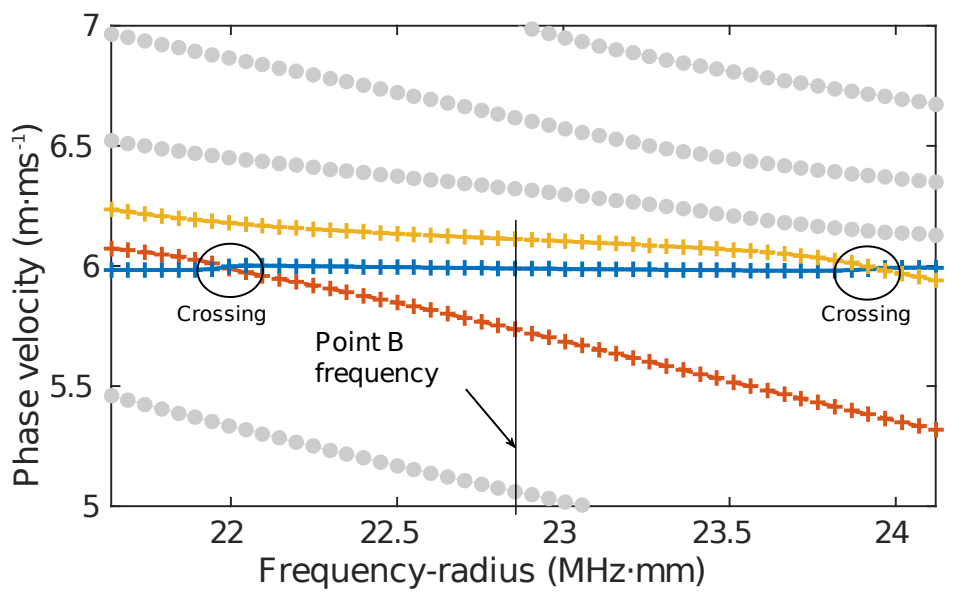

Figure 13: (Colour online) Crossing of phase velocity curves. Blue line: 12th leaky mode, red dashed line: 13th leaky mode, yellow dashed dotted line: 14th leaky mode. PML parameters: $d=h=a, \hat{\gamma}=1+2 \mathrm{j}$.

tering coefficients can be misleading. The post-processing of modal cross powers enables to identify the occurrence of strong interaction between modes (with no additional computational cost).

\subsubsection{Influence of PML modes on the solution in the very near-field}

Figure 14 shows the wavenumber spectrum computed at point $\mathrm{A}(f=$ $0.6 \mathrm{MHz} \cdot \mathrm{mm})$ in the complex half-plane $\operatorname{Re}(k a)>0$. The spectrum contains 13 leaky modes, including the fundamental L $(0,1)$ leaky mode, and 27 PML modes. Let us reduce the distance $\left|z_{i}-z_{\text {ref }}\right|$ to $0.25 a$ in order to reveal the contribution of PML modes on the solution in the very near-field of the notch.

Reflection and transmission coefficients are displayed in Fig. 15. They have been obtained by retaining either all the modes $\left(N_{\Sigma_{1}}=N_{\Sigma_{2}}=40\right)$, either leaky modes only $\left(N_{\Sigma_{1}}=N_{\Sigma_{2}}=13\right)$. It can be observed that the contribution of PML modes is necessary to accurately recover the results of Fig. 9 (particularly for the reflection coefficient).

The colormap of Fig. 14 displays the modulus of the modal coefficients of the transmitted modes retropropagated at $z=z_{\text {ref }}$, i.e. $\left|\alpha_{+m} \mathrm{e}^{+\mathrm{j} k_{+m}\left(z_{\mathrm{ref}}-z_{2}\right)}\right|$ $\times \sqrt{\left|\operatorname{Re}\left(\bar{P}_{+m}\right)\right|}$, for $h_{n} / a=0.8$ (similar colormaps can be obtained for other notch depths or in reflection). It shows that among PML modes, the modes with the highest attenuations $(\operatorname{Im}(k a))$ bear the highest modal contributions to the solution, which is a characteristic near-field behaviour. Therefore, the contribution of PML modes enables to improve the accuracy of results in the very near field. Their influence decreases as the distance to the notch increases, so that PML modes can eventually be neglected (as confirmed by Fig. 9).

Figure 14 also shows that another class of modes corresponding to highattenuation leaky modes can significantly contribute to the near field. Similarly 


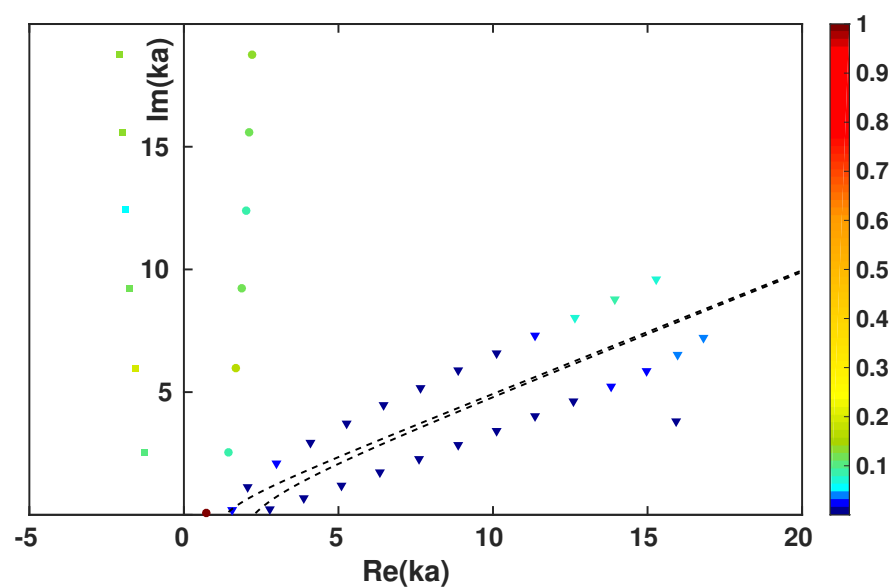

Figure 14: (Colour online) Wavenumber spectrum $\left(N_{\Sigma_{2}}=40\right)$ of a viscoelastic steel waveguide embedded into viscoelastic cement grout computed with the PML waveguide formulation at point $\mathrm{A}(f=0.6 \mathrm{MHz} \cdot \mathrm{mm})$. Circles: forward leaky modes, squares: backward leaky modes, triangles: PML modes. Colormap: modal coefficients of transmitted modes retropropagated at $z=z_{\text {ref }}$ for $h_{n} / a=0.8$, normalized by the maximum value. PML parameters: $d=h=$ $a, \hat{\gamma}=1+2 \mathrm{j}$.

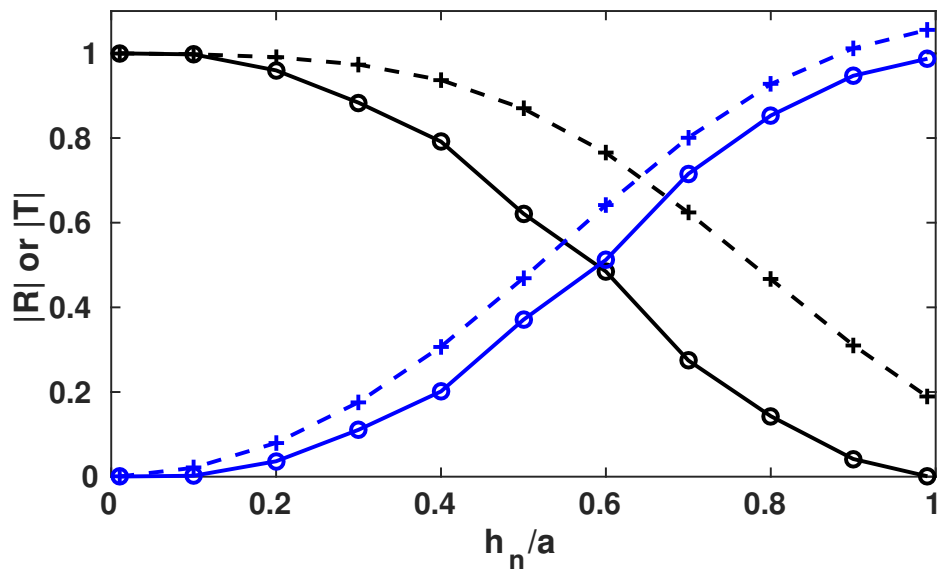

Figure 15: (Colour online) Scattering by a notch of varying depth at $f=0.6 \mathrm{MHz} \cdot \mathrm{mm}$ (point A) with $\left|z_{i}-z_{\text {ref }}\right|=0.25 a$. Dashed cross lines: $\left|R_{1,1}\right|$ (blue) and $\left|T_{1,1}\right|$ (black) with $N_{\Sigma_{1}}=$ $N_{\Sigma_{2}}=13$ (leaky modes only). Solid lines: $\left|R_{1,1}\right|$ (blue) and $\left|T_{1,1}\right|$ (black) with $N_{\Sigma_{1}}=N_{\Sigma_{2}}=$ 40. PML parameters: $d=h=a, \hat{\gamma}=1+2 \mathrm{j}$. 


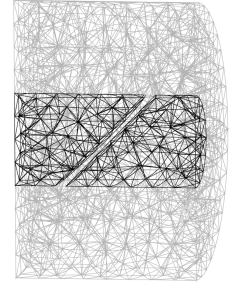

(a)
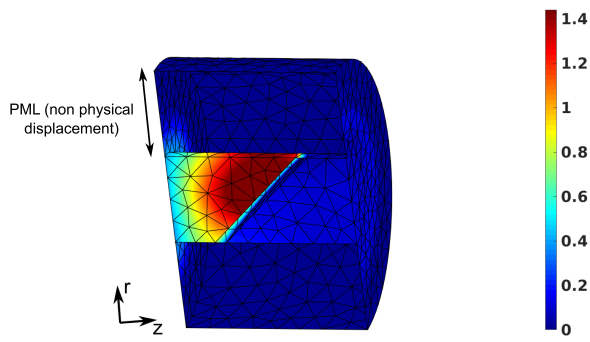

(b)

Figure 16: (Colour online) Oblique break of the core with an inclination of $45^{\circ}$. PML parameters are $d=a, h=2 a, \hat{\gamma}=2+4 \mathrm{j}$ for $\left|z_{i}-z_{\text {ref }}\right|=2 a$. (a) Finite element mesh (black: physical domain, grey: PML domain); (b) scattered field $\left|\mathbf{U}_{z} / a\right|$ at $f=0.6 \mathrm{MHz} \cdot \mathrm{mm}$.

to PML modes, these modes enable to reconstruct the wave fields near the inhomogeneity and have been included in the modal expansions. The sign of the wavenumber real part of high-attenuation leaky modes can be either positive or negative. The latter case $(\operatorname{Re}(k a)<0)$ corresponds to backward leaky modes $[39,52]$. As opposed to forward leaky modes, backward leaky modes decrease along the transverse direction (from a mathematical point of view, these modes are proper complex poles of the problem).

\subsection{Reflection by an oblique break}

The open waveguide, similar to the one considered in the previous sections, is now damaged by an oblique complete break of the core. The width of the break is set to $e_{n} / a=0.25$ and its angle of inclination varies. Contrary to the previous cases, the scattering problem is no longer axisymmetric and it must now be solved with the three-dimensional formulation of the hybrid method. The computational domain is depicted in Fig. 16a. Because of the symmetry of the problem, only half of the waveguide can be considered. The embedding medium is truncated with a PML of parabolic attenuation profile according to Eq. (32).

The problem is solved around point A between $0.4 \mathrm{MHz} \cdot \operatorname{mmand} 0.8 \mathrm{MHz} \cdot \mathrm{mm}$, which includes the fundamental longitudinal leaky mode $\mathrm{L}(0,1)$ and the fundamental flexural leaky mode $\mathrm{F}(1,1)$. The behaviour of the $\mathrm{F}(1,1)$ mode is more complex than the $\mathrm{L}(0,1)$ mode and the following PML parameters have been chosen in order to obtain acceptable results: $d=a, h=2 a, \hat{\gamma}=2+4 \mathrm{j}$. In the following, the scattering of both modes is studied for an incident $\mathrm{L}(0,1)$ leaky mode.

In order to limit the contribution of PML modes, the inlet and the outlet cross-sections boundaries, $\Sigma_{1}$ and $\Sigma_{2}$, are set at distances $\left|z_{i}-z_{\text {ref }}\right|=2 a$ (i.e. not too close to the break). The domain is meshed with 10 -nodes tetrahedral elements, yielding roughly 15000 dofs with 1290 dofs on each cross-section. The number of modes retained in the modal expansions has been set to $N_{\Sigma_{1}}=$ $N_{\Sigma_{2}}=100$. Numerical tests have shown that the contribution of higher order 


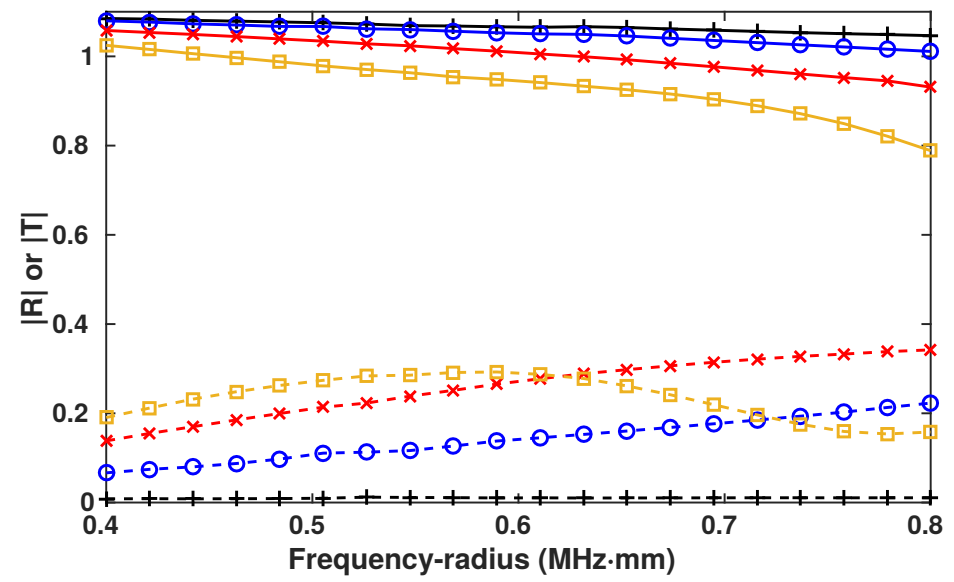

Figure 17: (Colour online) Scattering by an oblique break of the core for different inclination angles (three-dimensional problem). $\left|R_{\mathrm{L}(0,1), \mathrm{L}(0,1)}\right|: 0^{\circ}$ (dark cross line), $15^{\circ}$ (blue circle line), $30^{\circ}$ (red cross line), $45^{\circ}$ (yellow square line). $\left|R_{\mathrm{F}(1,1), \mathrm{L}(0,1)}\right|: 0^{\circ}$ (dark cross dashed line), $15^{\circ}$ (blue circle dashed line), $30^{\circ}$ (red cross dashed line), $45^{\circ}$ (yellow square dashed line).

modes on the results was negligible (as explained previously, more modes would have been necessary with cross-section boundaries closer to the inhomogeneity).

Figure 17 displays the reflection coefficients of both modes for four angles of inclination $\left(0^{\circ}, 15^{\circ}, 30^{\circ}\right.$ and $\left.45^{\circ}\right)$. The transmission coefficients, not shown here for conciseness, remain rather low.

For a break perpendicular to the waveguide axis (angle of $0^{\circ}$ ), the reflection coefficient of the $\mathrm{L}(0,1)$ leaky mode favourably matches with the axisymmetric notch test case (see Fig. 9 for $h_{n} / a=1$ ). Note that the value of the $\mathrm{F}(1,1)$ reflection coefficient is weak but non-null. According to the geometry, modal conversion into the flexural mode should not occur for an angle of $0^{\circ}$. This can be attributed to the moderate accuracy of flexural mode computation in the three-dimensional model (with additional computational cost, this could be improved either by mesh refinement or by setting a thicker PML).

When the angle increases from 0 to $30^{\circ}$, the reflection coefficient of the $\mathrm{L}(0,1)$ mode monotonically decreases while the reflection coefficient of the $\mathrm{F}(1,1)$ mode monotonically increases. As expected, the modal conversion from the longitudinal mode into the flexural mode becomes important as the angle increases. At $45^{\circ}$, the behaviour of the flexural mode is no longer monotonic and varies with the frequency. As an example, the corresponding scattered field is depicted in Fig. $16 \mathrm{~b}$ for $f=0.6 \mathrm{MHz} \cdot \mathrm{mm}$.

\section{Conclusion}

A PML-based hybrid method has been proposed for the numerical modelling of wave scattering by inhomogeneities in open waveguides. The modal represen- 
tation of the fields propagating in the undamaged part of the waveguide involves three types of modes: trapped modes, leaky modes and PML modes.

Comparisons with literature results have shown that the numerical method enables to obtain a good approximation of the scattering behaviour in open waveguides.

Based on the modal biorthogonality property, it has been shown that the modal cross power does not vanish in general in open waveguides. In particular, the modal cross-power can be significant when mode-crossing phenomena occur. This is likely to complicate the analysis of scattering phenomena.

A particular attention has been given to the influence of PML modes on the solution, and particularly on the scattering behaviour of leaky modes. In the very far-field, only the contribution of PML modes can accurately reconstruct the long-term diffraction of the field. Yet such a configuration is of less interest in the context of hybrid methods, the aim of which is to reduce the computation to the near-field of the inhomogeneity.

In the very near field, it has been shown that the contribution of PML modes can be not negligible and that leaky modes may be not sufficient to obtain accurate results. The weight of the PML mode contribution depends on the distance between the inhomogeneity and the transparent cross-section boundaries of the FE model. Therefore, the accuracy of the hybrid method has to be carefully checked by varying the distance of the boundaries and the number of modes involved in the modal expansions. For three-dimensional problems, it can be useful to set the transparent boundaries sufficiently far away from the inhomogeneity in order to avoid the computation of many PML modes.

As a final remark, the near-field behaviour of PML modes may raise feasibility issues for mode-matching methods when applied to open waveguides. Mode-matching methods are based on modal expansions set at the inhomogeneity position (see e.g. Ref. [53] in closed waveguides), which may require the computation of a high number of PML modes to achieve accurate results.

\section{Acknowledgements}

This work was supported by the Région Pays de la Loire.

\section{Appendix A. Axisymmetrical PML waveguide formulation}

Let us consider an axisymmetric waveguide. The formulation (1) is written in the cylindrical coordinate system $(\tilde{r}, \theta, z)$. The cross-section can be reduced to the radial direction $\tilde{r}$. The core of the waveguide has a radius $\tilde{r}=a$, and can be multilayered. Only axisymmetric solutions are considered, such that $\partial(.) / \partial \theta=0$ and $\tilde{u}_{\theta}=0$. Hence the displacement field in Eq. (1) is expressed as $\tilde{\mathbf{u}}=\left[\begin{array}{ll}\tilde{u}_{r} & \tilde{u}_{z}\end{array}\right]^{\mathrm{T}}$ and only depends on the variables $\tilde{r}$ and $z$. The strain field is given by $\tilde{\boldsymbol{\epsilon}}=\left[\begin{array}{llll}\tilde{\epsilon}_{r r} & \tilde{\epsilon}_{\theta \theta} & \tilde{\epsilon}_{z z} & 2 \tilde{\epsilon}_{r z}\end{array}\right]$. 
Using Eq. (2), the change of variable (3) is applied to Eq. (1). In the straindisplacement relation (13), the operators $\mathbf{L}_{S}$ and $\mathbf{L}_{z}$ are now given by:

$$
\mathbf{L}_{S}=\left(\frac{1}{\gamma}\left[\begin{array}{ll}
1 & 0 \\
0 & 0 \\
0 & 0 \\
0 & 1
\end{array}\right] \frac{\partial}{\partial r}+\left[\begin{array}{ll}
0 & 0 \\
1 & 0 \\
0 & 0 \\
0 & 0
\end{array}\right] \frac{1}{\tilde{r}}\right), \quad \mathbf{L}_{z}=\left[\begin{array}{ll}
0 & 0 \\
0 & 0 \\
0 & 1 \\
1 & 0
\end{array}\right] .
$$

Then, a one-dimensional FE discretization is applied along the radial direction, yielding the following interpolation on each element:

$$
\mathbf{u}^{e}(r, \omega)=\mathbf{N}_{\Sigma}^{e}(r) \boldsymbol{\Phi}^{e} .
$$

$\mathbf{N}_{\Sigma}^{e}(r)$ is the matrix of interpolation one-dimensional functions. $\boldsymbol{\Phi}^{e}(\omega)$ is the vector of nodal displacements. The final eigenproblem is identical to Eq. (14), where the element matrices are given by Eq.(15) with $\mathrm{d} S=2 \pi r \mathrm{~d} r$. Under the assumption used in this appendix, $\mathbf{C}$ is reduced to a four-by-four matrix. For instance, the matrix $\mathbf{C}$ for an isotropic material is:

$$
\mathbf{C}=\left[\begin{array}{cccc}
\lambda+2 \mu & \lambda & \lambda & 0 \\
\lambda & \lambda+2 \mu & \lambda & 0 \\
\lambda & \lambda & \lambda+2 \mu & 0 \\
0 & 0 & 0 & \mu
\end{array}\right]
$$

where $\lambda$ and $\mu$ are Lamé parameters.

\section{Appendix B. Total cross-section power flow} by:

The total-time averaged power flow across the whole cross-section is given

$$
\begin{aligned}
\Pi_{T} & =\operatorname{Re}\left(-\frac{\mathrm{j} \omega}{2} \mathbf{U}_{\Sigma}^{*} \mathbf{F}_{\Sigma}\right), \\
& =\frac{\mathrm{j} \omega}{4}\left(\mathbf{F}_{\Sigma}^{*} \mathbf{U}_{\Sigma}-\mathbf{U}_{\Sigma}^{*} \mathbf{F}_{\Sigma}\right) .
\end{aligned}
$$

Considering the modal expansions of the fields $\mathbf{U}_{\Sigma}$ and $\mathbf{F}_{\Sigma}$, one gets:

$$
\begin{aligned}
\Pi_{T} & =\frac{j \omega}{4}\left(\sum_{n=-N}^{N} \alpha_{n}^{*} \mathbf{F}_{n}^{*} \sum_{m=-N}^{N} \alpha_{m} \mathbf{U}_{m}-\sum_{n=-N}^{N} \alpha_{n}^{*} \mathbf{U}_{n}^{*} \sum_{m=-N}^{N} \alpha_{m} \mathbf{F}_{m}\right), \\
& =\frac{\mathrm{j} \omega}{4}\left(\sum_{m=-N}^{N}\left|\alpha_{m}\right|^{2}\left(\mathbf{F}_{m}^{*} \mathbf{U}_{m}-\mathbf{U}_{m}^{*} \mathbf{F}_{m}\right)+\sum_{m=-N}^{N} \sum_{n \neq m} \alpha_{n}^{*} \alpha_{m}\left(\mathbf{F}_{n}^{*} \mathbf{U}_{m}-\mathbf{U}_{n}^{*} \mathbf{F}_{m}\right)\right) .
\end{aligned}
$$

From the above equation, the modal cross-power $P_{m, n}=\frac{\mathrm{j} \omega}{4}\left(\mathbf{F}_{n}^{*} \mathbf{U}_{m}-\mathbf{U}_{n}^{*} \mathbf{F}_{m}\right)$ can be identified. For $m=n$, it can be checked that $P_{m, m}=\frac{\omega}{2} \operatorname{Im}\left(\mathbf{U}_{m}^{*} \mathbf{F}_{m}\right)=$ 
$\operatorname{Re}\left(P_{m}\right)$. Finally, the total-time averaged power flow can then be written:

$$
\Pi_{T}=\sum_{m=-N}^{N}\left|\alpha_{m}\right|^{2} \operatorname{Re}\left(P_{m}\right)+\sum_{m=-N}^{N} \sum_{n \neq m} \alpha_{n}^{*} \alpha_{m} P_{m, n} .
$$

\section{Appendix C. Filtering of PML modes}

It can be necessary to filter out PML modes, e.g. for the visualization of dispersion curves, in which only leaky and trapped modes are of interest. In this paper, we use the filtering criterion proposed in Ref. [33]. It is based on the ratio of the imaginary part over the modulus of the kinetic energy, defined from Eq. (25) but integrating the whole cross-section (i.e. including the PML, so that the overbar has to be discarded in Eq. (25)). The modes are retained if they fulfil the following criterion:

$$
1-\frac{\operatorname{Im}\left(T_{m}\right)}{\left|T_{m}\right|}>\eta_{\min }
$$

where $0<\eta_{\min }<1$ is a user-defined parameter.

[1] B. N. Pavlakovic, M. J. S. Lowe, P. Cawley, High-frequency low-loss ultrasonic modes in imbedded bars, J. Appl. Mech. 68 (1) (2001) 67-75. doi:10.1115/1.1347995.

[2] L. Laguerre, F. Treyssede, Non destructive evaluation of seven-wire strands using ultrasonic guided waves, Eur. J. Environ. Civ. Eng. 15 (4) (2011) 487-500. doi:10.1080/19648189.2011.9693342.

[3] M. D. Beard, M. J. S. Lowe, Non-destructive testing of rock bolts using guided ultrasonic waves, Int. J. Rock Mech. Mining Sci. 40 (4) (2003) 527536. doi:10.1016/S1365-1609(03)00027-3.

[4] M. D. Beard, M. J. S. Lowe, P. Cawley, Ultrasonic guided waves for inspection of grouted tendons and bolts, J. Mat. Civil Eng. 15 (3) (2003) 212-218. doi:10.1061/(ASCE)0899-1561(2003)15:3(212).

[5] E. Leinov, M. J. S. Lowe, P. Cawley, Investigation of guided wave propagation in pipes fully and partially embedded in concrete, J. Acoust. Soc. Am. 140 (6) (2016) 4528-4539. doi:10.1121/1.4972118.

[6] T. Vogt, M. Lowe, P. Cawley, The scattering of guided waves in partly embedded cylindrical structures, J. Acoust. Soc. Am. 113 (3) (2003) 12581272. doi:10.1121/1.1553463.

[7] W. Duan, R. Kirby, P. Mudge, On the scattering of torsional waves from axisymmetric defects in buried pipelines, J. Acoust. Soc. Am. 141 (5) (2017) 3250-3261. arXiv:http://dx.doi.org/10.1121/1.4983192, doi:10.1121/1.4983192. 
[8] M. Gallezot, F. Treyssède, L. Laguerre, A numerical method for the scattering by defects in axisymmetrical open elastic waveguides, Proc. Engrg. 199 (2017) 1527-1532. doi:10.1016/j.proeng.2017.09.495.

[9] V. Baronian, A. S. Bonnet-Ben Dhia, E. Luneville, Transparent boundary conditions for the harmonic diffraction problem in an elastic waveguide, J. Comp. Appl. Math. 234 (6, SI) (2010) 1945-1952. doi:10.1016/j.cam.2009.08.045.

[10] F. Benmeddour, F. Treyssède, L. Laguerre, Numerical modeling of guided wave interaction with non-axisymmetric cracks in elastic cylinders, Int. J. Solid Struct. 48 (5) (2011) 764-774. doi:10.1016/j.ijsolstr.2010.11.013.

[11] F. Treyssède, Mode propagation in curved waveguides and scattering by inhomogeneities: Application to the elastodynamics of helical structures, J. Acoust. Soc. Am. 129 (4) (2011) 1857-1868. doi:10.1121/1.3559682.

[12] Y. H. Cho, J. L. Rose, A boundary element solution for a mode conversion study on the edge reflection of lamb waves, J. Acoust. Soc. Am. $99(4,1)$ (1996) 2097-2109. doi:10.1121/1.415396.

[13] N. Rattanawangcharoen, W. Zhuang, A. H. Shah, S. K. Datta, Axisymmetric guided waves in jointed laminated cylinders, J. Eng. Mech. 123 (10) (1997) 1020-1026. doi:10.1061/(ASCE)0733-9399(1997)123:10(1020).

[14] J. M. Galan, R. Abascal, Numerical simulation of lamb wave scattering in semi-infinite plates, Int. J. Num. Meth. Eng. 53 (5) (2002) 1145-1173. doi:10.1002/nme.331.

[15] W. Duan, R. Kirby, P. Mudge, On the scattering of elastic waves from a non-axisymmetric defect in a coated pipe, Ultrasonics 65 (2016) 228-241. doi:10.1016/j.ultras.2015.09.019.

[16] A.-S. B.-B. Dhia, C. Chambeyron, G. Legendre, On the use of perfectly matched layers in the presence of long or backward propagating guided elastic waves, Wave Motion 51 (2) (2014) 266-283. doi:10.1016/j.wavemoti.2013.08.001.

[17] R. N. Thurston, Elastic waves in rods and clad rods, J. Acoust. Soc. Am. 64 (1) (1978) 1-37. doi:10.1121/1.381962.

[18] D. Marcuse, Theory of Dielectric Optical Waveguides, Academic Press, 1991.

[19] P. Malischewsky, Surface Waves and Discontinuities, Elsevier, 1987.

[20] R. E. Collin, Field theory of guided waves, IEEE Press, Wiley, 1991. 
[21] V. Maupin, The radiation modes of a vertically varying halfspace: a new representation of the complete Green's function in terms of modes, Geophys. Int. 126 (3) (1996) 762-780. arXiv:http://gji.oxfordjournals.org/content/126/3/762.full.pdf + html, doi:10.1111/j.1365-246X.1996.tb04701.x.

[22] J. Hu, C. R. Menyuk, Understanding leaky modes: slab waveguide revisited, Advances in Optics and Photonics 1 (1) (2009) 58-106. doi:10.1364/AOP.1.000058.

[23] J. M. de Oliveira Barbosa, J. Park, E. Kausel, Perfectly matched layers in the thin layer method, Comput. Meth.Appl. Mech. Engrg. 217 (2012) 262-274. doi:10.1016/j.cma.2011.12.006.

[24] F. Treyssède, K. L. Nguyen, A.-S. Bonnet-BenDhia, C. Hazard, Finite element computation of trapped and leaky elastic waves in open stratified waveguides, Wave Motion 51 (7) (2014) 1093-1107. doi:10.1016/j.wavemoti.2014.05.003.

[25] K. L. Nguyen, F. Treyssède, C. Hazard, Numerical modeling of threedimensional open elastic waveguides combining semi-analytical finite element and perfectly matched layer methods, J. Sound Vib. 344 (2015) 158-178. doi:10.1016/j.jsv.2014.12.032.

[26] P. Zuo, X. Yu, Z. Fan, Numerical modeling of embedded solid waveguides using SAFE-PML approach using a commercially available finite element package, NDT \& E International 90 (2017) 11-23. doi:10.1016/j.ndteint.2017.04.003.

[27] H. Gravenkamp, C. Birk, C. Song, Computation of dispersion curves for embedded waveguides using a dashpot boundary condition, J. Acoust. Soc. Am. 135 (3) (2014) 1127-1138. doi:10.1121/1.4864303.

[28] M. Mazzotti, I. Bartoli, A. Marzani, E. Viola, A coupled SAFE-2.5D BEM approach for the dispersion analysis of damped leaky guided waves in embedded waveguides of arbitrary cross-section, Ultrasonics 53 (7) (2013) 1227-1241. doi:10.1016/j.ultras.2013.03.003.

[29] A. V. Astaneh, M. N. Guddati, Dispersion analysis of composite acoustoelastic waveguides, Composites Part B: Engineering 130 (2017) 200-216. doi:10.1016/j.compositesb.2017.07.040.

[30] M. Castaings, M. Lowe, Finite element model for waves guided along solid systems of arbitrary section coupled to infinite solid media, J. Acoust. Soc. Am. 123 (2) (2008) 696-708. doi:10.1121/1.2821973.

[31] T. Hayashi, D. Inoue, Calculation of leaky lamb waves with a semianalytical finite element method, Ultrasonics 54 (6) (2014) 1460-1469. doi:10.1016/j.ultras.2014.04.021. 
[32] S. Kim, J. E. Pasciak, The computation of resonances in open systems using a perfectly matched layer, Math. Comp. 78 (267) (2009) 1375-1398. doi:10.1090/S0025-5718-09-02227-3.

[33] M. Gallezot, F. Treyssède, L. Laguerre, A modal approach based on perfectly matched layers for the forced response of elastic open waveguides, J. Comp. Phys. 356 (2018) 39-409. doi:10.1016/j.jcp.2017.12.017.

[34] W. C. Chew, W. H. Weedon, A 3D perfectly matched medium from modified maxwells equations with stretched coordinates, Microwave and Optical Technology Letters 7 (13) (1994) 599-604. doi:10.1002/mop.4650071304.

[35] F. Tisseur, K. Meerbergen, The quadratic eigenvalue problem, SIAM Rev. 43 (2) (2001) 235-286. doi:10.1137/S0036144500381988.

[36] F. Treyssède, L. Laguerre, Numerical and analytical calculation of modal excitability for elastic wave generation in lossy waveguides, J. Acoust. Soc. Am. 133 (6) (2013) 3287-3837. doi:10.1121/1.4802651.

[37] A. Bernard, M. J. S. Lowe, M. Deschamps, Guided waves energy velocity in absorbing and non-absorbing plates, J. Acoust. Soc. Am. 110 (1) (2001) 186-196. doi:10.1121/1.1375845.

[38] B. A. Auld, Acoustic fields and waves in solids, Vol. 2, A Wiley-Interscience publication, 1973.

[39] I. A. Nedospasov, V. G. Mozhaev, I. E. Kuznetsova, Unusual energy properties of leaky backward lamb waves in a submerged plate, Ultrasonics 77 (2017) 95-99. doi:10.1016/j.ultras.2017.01.025.

[40] M. V. Predoi, M. Castaings, L. Moreau, Influence of material viscoelasticity on the scattering of guided waves by defects, J. Acoust. Soc. Am. 124 (5) (2008) 2883-2894. doi:10.1121/1.2977604.

[41] B. Hosten, L. Moreau, M. Castaings, Reflection and transmission coefficients for guided waves reflected by defects in viscoelastic material plates, J. Acoust. Soc. Am. 121 (6) (2007) 3409-3417. doi:10.1121/1.2723652.

[42] A. Gunawan, S. Hirose, Mode-exciting method for lamb wavescattering analysis, J. Acoust. Soc. Am. 115 (3) (2004) 996-1005. doi:10.1121/1.1639330.

[43] R. Sammut, A. W. Snyder, Leaky modes on a dielectric waveguide: orthogonality and excitation, Applied Optics 15 (4) (1976) 1040-1044. doi:10.1364/AO.15.001040.

[44] A. W. Snyder, Coupled-mode theory for optical fibers, J. Opt. Soc. Am. 62 (11) (1972) 1267-1277. doi:10.1364/JOSA.62.001267. 
[45] D. F. Williams, F. Olyslager, Modal cross power in quasi-TEM transmission lines, IEEE Microwave and Guided Wave Letters 6 (11) (1996) 413-415. doi: $10.1109 / 75.541457$.

[46] R. B. Lehoucq, D. C. Sorensen, C. Yang, ARPACK users' guide: solution of large-scale eigenvalue problems with implicitly restarted Arnoldi methods, Vol. 6, Siam, 1998.

[47] F. Olyslager, Discretization of continuous spectra based on perfectly matched layers, SIAM J. Appl. Math. 64 (4) (2004) 1408-1433. doi:10.1137/S0036139903430197.

[48] B. N. Pavlakovic, Leaky guided ultrasonic waves in ndt, Ph.D. thesis, University of London - Imperial College of Science, Technology and Medicine (1998).

[49] M. D. Beard, M. J. S. Lowe, P. Cawley, Inspection of steel tendons in concrete using guided waves, Rev. Quantitative Nondestruct. Eval. 22 (2003) $1139-1147$.

[50] C. W. Chan, P. Cawley, Lamb waves in highly attenuative plastic plates, J. Acoust. Soc. Am. 104 (2) (1998) 874-881. doi:10.1121/1.423332.

[51] E. Manconi, S. Sorokin, On the effect of damping on dispersion curves in plates, Int. J. Solid Struct. 50 (11-12) (2013) 1966-1973. doi:10.1016/j.ijsolstr.2013.02.016.

[52] T. Tamir, A. A. Oliner, Guided complex waves. part 1: Fields at an interface, Proc. IEEE 110 (2) (1963) 310-324. doi:10.1049/piee.1963.0044.

[53] E. Le Clézio, M. Castaings, B. Hosten, The interaction of the S0 Lamb mode with vertical cracks in an aluminum plate, Ultrasonics 40 (2002) 187-192. doi:10.1016/S0041-624X(02)00135-X. 